\title{
Quality Meshing for Polyhedra with Small Angles*
}

\author{
Siu-Wing Cheng ${ }^{\dagger} \quad$ Tamal K. Dey $\quad$ Edgar A. Ramos ${ }^{\ddagger} \quad$ Tathagata Ray $^{\ddagger}$
}

April 11, 2005

\begin{abstract}
We present an algorithm to compute a Delaunay mesh conforming to a polyhedron possibly with small input angles. The radius-edge ratio of most output tetrahedra are bounded by a constant, except possibly those that are provably close to small angles. Furthermore, the mesh is not unnecessarily dense in the sense that the edge lengths are at least a constant fraction of the local feature sizes at the edge endpoints. This algorithm is simple to implement as it eliminates most of the computation of local feature sizes and explicit protective zones. Our experimental results validate that few skinny tetrahedra remain and they lie close to small acute input angles.
\end{abstract}

\section{Introduction}

The need for meshing a polyhedral domain with well shaped tetrahedra arises as an important problem in finite element methods. Mainly there are two approaches known for the problem, octree based refinement $[1,13]$ and the Delaunay refinement $[7,9,11,12,16,17]$. Among these two, often the latter is preferred for its directional independence and better quality meshing in general.

The Delaunay refinement techniques developed with the work of Chew [6] and Ruppert [16] who showed how to mesh a polygonal domain in $2 \mathrm{D}$ with quality triangles. Shewchuk extended the technique to polyhedral domains in three dimensions [17]. This extension has two shortcomings. First, it guarantees bounded radius-edge ratio but not bounded aspect ratio. The radius-edge ratio of a tetrahedron is the ratio of its circumradius to its smallest edge length. Bounded radius-edge ratio eliminates all kinds of poor tetrahedra but not slivers. Second, the Delaunay refinement works only with input domains where no angle is smaller than $\frac{\pi}{2}$. The sliver exudation method of Cheng et al. [4] addressed the sliver problem though for unbounded domains. Chew [7] and $\mathrm{Li}$ and Teng [11] proposed non-deterministic point placement to eliminate slivers for bounded domains. Cheng and Dey [3] combined the sliver exudation method with the Delaunay refinement to give a deterministic algorithm for meshing bounded domains with tetrahedra having bounded aspect ratio.

\footnotetext{
${ }^{*}$ Research is supported by RGC, Hong Kong, China, under grants HKUST6088/99E, HKUST6190/02E, Army Research Office, USA under grant DAAD19-02-1-0347 and NSF, USA under grants DMS-0310642 and CCR-0430735.

${ }^{\dagger}$ Department of Computer Science Science, HKUST, Hong Kong. Email: scheng@cs.ust.hk

${ }^{\ddagger}$ Department of Computer Science and Engineering, Ohio State University, Ohio, USA. Email: tamaldey@cis.ohio-state.edu and rayt@cis.ohio-state.edu

${ }^{\S}$ Department of Computer Science, University of Illinois at Urbana-Champaign, Urbana-Champaign, USA. Email: eramosn@cs.uiuc.edu
} 
However, these works did not address the problem of small angles, specifically input angles less than $\frac{\pi}{2}$. Shewchuk [18] addressed the problem of small angles with constrained Delaunay triangulation though without any guarantees about the shape quality of the tetrahedra. The question of handling small angles with Delaunay refinement was left open. Murphy, Mount and Gable [14] and Cohen-Steiner, de Verdière and Yvinec [8] presented algorithms that can triangulate any polyhedral domain with Delaunay tetrahedra though without any quality guarantee. Recently, Cheng and Poon [5] proposed a Delaunay meshing algorithm that can handle small angles with quality guarantees. The output mesh is graded, i.e., for each mesh vertex $v$, its nearest neighbor distance is $\Omega(f(v))$ where $f(v)$ is the local feature size at $v$. The radius-edge ratio of all tetrahedra are bounded by a constant. The constant depends on the smallest input angle in the vicinity of input edges, but it is independent of the input domain elsewhere. Although this algorithm is a significant theoretical progress for dealing with small angles, its practical viability is doubtful. The main reason is that it constructs a protecting region (a union of balls) around each input edge explicitly, which is complicated and time intensive. This construction involves computing a feature related distance for a number of points on the edges, intersections among spheres and input facets, and intersections among spheres. Moreover, the subsequent refinement has to deal with curved edges and spherical surfaces.

In this paper we present a new algorithm for meshing polyhedra which has similar guarantees as that of [5], but it is much simpler to implement. Our algorithm produces a graded Delaunay mesh. The radius-edge ratio of most output tetrahedra are bounded by a constant. For any remaining tetrahedron of poor shape, all of its vertices are provably close to some small input angle. In this new algorithm, only input vertices and edges at acute input angles are protected. More importantly, we get rid of the explicit construction of any protecting region, and limit the computation of local feature sizes at the input vertices only. Another novelty is that we employ the splitting of non-Delaunay triangles in recovering the input boundary. Earlier works, instead, relied on splitting non-Gabriel triangles, that is, the triangles whose smallest circumscribing balls are not empty of vertices. Our experience is that the latter approach may cause many more points to be inserted. The splitting of non-Delaunay triangles is also essential as we do not have an explicit protecting region. We have implemented our algorithm and we present experimental results to demonstrate that our algorithm indeed handles polyhedra with small angles. The results validate our claim on the quality of the output tetrahedra and show that few skinny tetrahedra remain.

\section{Preliminaries}

Basic definitions. We need the following definitions most of which have been introduced in earlier works.

Skinny tetrahedra. Let $R, \ell$ be the circumradius and shortest edge length respectively of a tetrahedron $\tau$. Let $\rho(\tau)=R / \ell$. We say $\tau$ is $\rho_{0}$-skinny or simply skinny if $\rho(\tau)>\rho_{0}$.

Input domain. A vertex $v$ is a point in $\mathbb{R}^{3}$; its boundary $\partial v$ is $v$ itself. An edge $e$ is a line segment joining two vertices, say $v_{1}$ and $v_{2}$. The boundary $\partial e$ is $\left\{v_{1}, v_{2}\right\}$. A facet is a subset of a plane in $\mathbb{R}^{3}$ bounded by a collection of simple polygonal cycles, i.e., for a facet $F, \partial F$ is a collection of edges and vertices forming polygonal cycles. A piecewise linear complex PLC is a collection of elements which are vertices, edges and facets that form a complex in the following sense. The intersection of any two elements is either empty or is a collection of lower dimensional elements 
on their boundaries. We assume that the input polyhedron is a subset of $\mathbb{R}^{3}$ bounded by a 2-manifold which is the underlying space of a PLC.

Two adjacent facets $F_{1}$ and $F_{2}$ may or may not be coplanar. The intersection $F_{1} \cap F_{2}$ may consist of several connected components. A component may be a single vertex, a single edge, or a polygonal chain of edges. In the case of a single vertex, we call it an isolated vertex. If $F_{1}$ and $F_{2}$ are non-coplanar, the components in $F_{1} \cap F_{2}$ are collinear and they lie along the intersection line of the support planes of $F_{1}$ and $F_{2}$.

The requirement that any two elements can intersect only at their boundaries disallows special cases such as isolated vertex or a dangling edge in the middle of a facet. We believe that, after adding extra steps to deal with special cases, our algorithm can be extended to handle any polyhedral domain as long as the union of the elements in the input PLC form a 2-manifold without boundary.

To construct the mesh, we bound this domain in a large enough rectangular box, mesh the inside of the box conforming to the input polyhedron, and only keep the tetrahedra covering the interior of the input polyhedron. The introduction of a bounding box has been used before $[10,16]$. Let $\mathcal{B}$ denote the boundary of the bounding box. We use $\mathcal{P}$ to denote the PLC of the input polyhedron together with $\mathcal{B}$. For convenience, sometimes we abuse the notation $\mathcal{P}$ to denote the underlying space of this input PLC.

Incident and adjacent elements. Let $\partial F$ denote the boundary of an element $F \in \mathcal{P}$. We say that an element $F \in \mathcal{P}$ is incident to an element $G \in \mathcal{P}$ if either $F \subseteq \partial G$ or $G \subseteq \partial F$. Two elements of $\mathcal{P}$ are adjacent if their intersection is non-empty.

Local feature size. The local feature size for $\mathcal{P}$ is a function $f: \mathbb{R}^{3} \rightarrow \mathbb{R}$ where $f(x)$ is the radius of the smallest ball centered at $x$ intersecting two non-adjacent elements of $\mathcal{P}$.

Input angles. We define different types of angle at the vertices, edges and facets of $\mathcal{P}$. There are two types of input angles at each vertex $u$ of $\mathcal{P}$. For any two incident edges of $u$, we measure the angle between them. We call such angles edge-edge angles. For any edge $u v$ and a facet $F$ incident to $u$ such that $u v$ is neither incident on $F$ nor coplanar with $F$, the angle between $u v$ and $F$ is $\min \{\angle p u v: p \in F, p \neq u\}$. We call such angles edge-facet angles. Note that $u v$ and $F$ are required to be non-coplanar because the angle between $u v$ and $F$ can be zero otherwise. This happens when the ray emitting from $u$ through $v$ intersects $F$ at another point. At an edge of $\mathcal{P}$, we measure the internal and external dihedral angles at the edge.

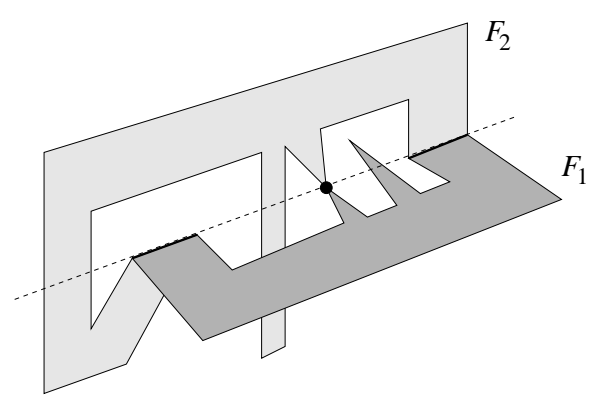

Figure 1: There are two edges and one isolated vertex in $F_{1} \cap F_{2}$.

The remaining angles involve two adjacent non-coplanar facets $F_{1}$ and $F_{2}$. Let $H_{1}$ and $H_{2}$ 
be the support planes of $F_{1}$ and $F_{2}$, respectively. Notice that $F_{i}$ may cross the line $H_{1} \cap H_{2}$ and the intersection of $F_{1}$ and $F_{2}$ may be disconnected; see Figure 1 . The planes $H_{1}$ and $H_{2}$ divide $\mathbb{R}^{3}$ into four closed regions. We call a region a wedge of $F_{1}$ and $F_{2}$ if it contains points on both $F_{1}$ and $F_{2}$ other than those on the line $H_{1} \cap H_{2}$. The dihedral angle subtended by the wedge at the line $H_{1} \cap H_{2}$ is called its wedge angle. Notice that wedges and wedge angle are well defined only when $F_{1}$ and $F_{2}$ are non-coplanar. It is essential to distinguish between wedges and non-wedges among the four closed regions induced by $H_{1}$ and $H_{2}$. Suppose that we do not and consider the dihedral angles of all four regions as input angles instead. Then even a very round convex polyhedron with almost equilateral triangular facets might contain input angles arbitrarily close to zero (when the internal dihedral angle of two adjacent facets is arbitrarily close to $\pi$ ). This is against the intuition that only the vertex angles of the facets matter in this case.

Throughout this paper, we use $\alpha_{m}$ to denote the minimum input angle in $\mathcal{P}$. Notice that $\alpha_{m}$ is strictly positive according to our definitions. We will need the following lemma about the angles between a line segment and a plane that it is attached to.

Lemma 2.1 Let $a b$ be a line segment. Let $H$ be a plane that contains $b$. Let $a^{\prime}$ be the orthogonal projection of a onto $H$. Then for any point $x \in H$ other than $b, \cos \angle a b x=\cos \angle a b a^{\prime} \cdot \cos \angle a^{\prime} b x$.

Proof. Let $i, j$, and $k$ be three orthonormal vectors with origin at $b$ such that $i$ lies along $a^{\prime} b$, $j$ is normal to $H$ and $j \cdot \overrightarrow{b a} \geq 0$, and $k \cdot \overrightarrow{b x} \geq 0$. Then

$$
\begin{aligned}
\cos \angle a b x & =\frac{(a-b)}{\|a-b\|} \cdot \frac{(x-b)}{\|x-b\|} \\
& =\left(i \cos \angle a b a^{\prime}+j \sin \angle a b a^{\prime}\right) \cdot\left(i \cos \angle a^{\prime} b x+k \sin \angle a^{\prime} b x\right) \\
& =\cos \angle a b a^{\prime} \cdot \cos \angle a^{\prime} b x .
\end{aligned}
$$

Lemma 2.1 implies that if we rotate a ray $\overrightarrow{b x}$ around $b$ in $H$ starting from the direction of $a^{\prime}-b$, the angle between $\overrightarrow{b a}$ and $\overrightarrow{b x}$ increases continuously until the ray reaches the direction of $b-a^{\prime}$. After that the angle decreases continuously until it returns to the direction of $a^{\prime}-b$. See Figure 2.

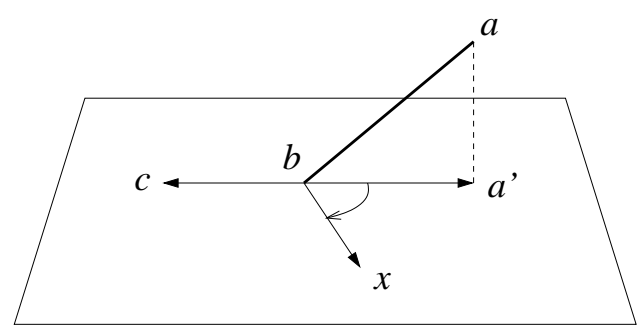

Figure 2: As the ray $\overrightarrow{b x}$ rotates a full circle in clockwise order starting from $b \vec{a}^{\prime}, \angle a b x$ strictly increases as $x$ goes from $a^{\prime}$ to $c$, and then strictly decreases as it goes from $c$ to $a^{\prime}$.

Sharp features. We call an edge sharp if the internal or external dihedral angle at the edge is acute. Sharp edges can be identified by taking the dot product of the outward normals of their incident facets. If the dot product is negative, the edge is sharp. 
We call a vertex $u$ sharp if an edge-edge angle or an edge-facet angle at $u$ is acute, or if $u$ is an endpoint of a sharp edge. Two edges $u v$ and $u w$ form an acute angle if the dot product $(v-u) \cdot(w-u)$ is positive. Checking if an edge $u v$ forms an acute angle with an incident facet $F$ of $u$ requires a bit more work. We first check that $u v$ is not incident to $F$ and $u v$ is not coplanar with $F$. Then the angle between $u v$ and $F$ is acute if and only if $\angle v u w$ is acute for some vertex $w$ of $F$.

We call a wedge sharp if its wedge angle is acute. Our algorithm only needs to identify sharp vertices and sharp edges. That is, our algorithm only works with edge-edge angles, edge-facet angles, and dihedral angles. The wedges, wedge angles, and the minimum input angle $\alpha_{m}$ are used in the analysis only.

Notice that, according to our assumption no angle of the bounding box $\mathcal{B}$ is less than $\frac{\pi}{2}$ though other input angles in $\mathcal{P}$ may be so.

\section{Algorithm}

The algorithm has three distinct phases, an initialization phase called INITIALIZE, a conforming phase called Conform and a refinement phase called REFine. In Initialize we protect the sharp vertices and compute the initial Delaunay triangulation. In CONFORM, we first refine edges and facets so that the 3D Delaunay triangulation conforms to $\mathcal{P}$. Then, we determine certain protecting balls around sharp edges. Some points are disallowed to be inserted in the REFINE phase in these protecting balls and the vertex balls around sharp vertices. Notice that there is no explicit construction of a protecting region. After Conform, Refine starts splitting skinny tetrahedra which may trigger more splitting of the edges and facets. The algorithm maintains a vertex set $\mathcal{V}$ which is updated along with its Delaunay triangulation Del $\mathcal{V}$ as more vertices are generated.

At any generic step of the algorithm, the vertices on any edge of $\mathcal{P}$ divide it into subsegments. The subsegments inside the vertex balls are protected by the vertex balls, so we are not concerned about them. We call a subsegment sharp if it lies outside the vertex balls and on a sharp edge. It is non-sharp otherwise. We define a circumball of a subsegment to be a finite ball with the subsegment endpoints on its boundary. The diametric ball of a subsegment is its smallest circumball. A point $p$ encroaches a subsegment if $p$ is not an endpoint of the subsegment and lies on or inside its diametric ball. See Figure 3(a). This definition of subsegment encroachment is stricter than the usual one as it includes the case where $p$ lies on the ball boundary. This helps to simplify proofs later.

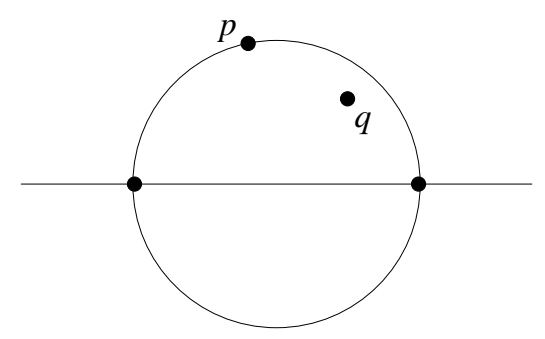

(a)

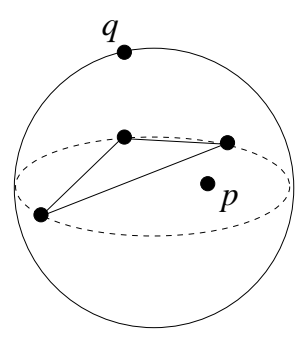

(b)

Figure 3: In Figure (a), $p$ and $q$ encroach the subsegment. In Figure (b), $p$ encroaches the subfacet but $q$ does not. 
When no subsegment is encroached by any vertex in $\mathcal{V}$, the facets are decomposed into subfacets defined as follows. For each facet $F$ of $\mathcal{P}$, consider the 2D Delaunay triangulation of the vertices in $\mathcal{V} \cap F$. The Delaunay triangles in $F$ are its subfacets. Thanks to the stricter definition of subsegment encroachment, the circumcenters of subfacets lie strictly in the interior of $F$, see Cheng and Dey [3]. We define a circumball of a subfacet $h$ to be a finite ball with the vertices of $h$ on its boundary. The diametric ball of $h$ is its smallest circumball. A point $p$ encroaches $h$ if $p$ lies inside its diametric ball. Notice that we cannot use the stricter definition of encroachment as in the subsegment case because more than three vertices in $\mathcal{V} \cap F$ may lie on the same empty circle.

There are several subroutines used by our algorithm. We describe them in Sections 3.1-3.5 and then give the algorithm in Section 3.6.

\subsection{Sharp vertex protection}

First, we protect the sharp vertices with empty protecting balls called vertex balls. Points are disallowed to be inserted inside these vertex balls at later stages. This will mean that certain skinny tetrahedra are not removed since their removal causes insertions of vertices inside these vertex balls. Because of this constraint we compute the feature sizes at the sharp vertices explicitly and use it to compute the vertex balls. This allows us to argue that the skinny tetrahedra that we left out lie near the sharp vertices and edges of the input.

For each sharp vertex $u$, we compute its distance from all elements of $\mathcal{P}$ which are not incident to $u$. This distance is the local feature size $f(u)$ at $u$. A brute-force computation of the feature size takes $O(n)$ time per vertex where $n$ is the number of elements in $\mathcal{P}$. This brute-force computation is not prohibitive in practice since it is performed only once and that too before splitting any elements of the input $\mathcal{P}$.

We put a vertex ball $\widehat{u}$ with radius $f(u) / 4$. (In the rest of this paper, we use $\widehat{x}$ to denote a ball centered at a point $x$. When $x$ is a vertex of $\mathcal{P}, \widehat{x}$ refers to its vertex ball.) The points where the boundary of $\widehat{u}$ intersects edges of $\mathcal{P}$ are inserted into the vertex set $\mathcal{V}$. We protect a subset of $\widehat{u} \cap \mathcal{P}$ using the method of Cohen-Steiner, de Verdière and Yvinec [8]. At any generic step of the algorithm, $\mathcal{V}$ contains vertices on the arc where a facet $F$ incident to $u$ intersects the boundary of $\widehat{u}$. The segments connecting consecutive points on such an arc form shield subsegments. Let $a b$ be a shield subsegment. If the angle of the circular sector $a u b$ on $F$ is at least $\pi$ or $a b$ is encroached, it is split by the following method called SOS according to Cohen-Steiner et al. [8] (see [2] for a more general version of this strategy). If the angle of the sector $a u b$ on $F$ is at least $\pi$, we insert the midpoint $x$ on the arc between $a$ and $b$ on the boundary of $\widehat{u} \cap F$. The subsegment $a b$ is replaced with two shield subsegments $a x$ and $b x$; see Figure 4(a). So this type of splitting happens at most once for $u$, in which case $a$ and $b$ lie on the boundary edges of $F$ incident to $u$. If the angle of the sector $a u b$ is less than $\pi$ and $a b$ is encroached, we insert the midpoint $x$ on the shorter arc between $a$ and $b$ on the boundary of $\widehat{u}$. The subsegment $a b$ is replaced with two shield subsegments $a x$ and $b x$; see Figure 4(b).

When no shield subsegment corresponds to a sector at $u$ with angle $\pi$ or more, the shield subsegments around $u$ create a set of shield subfacets incident to $u$. Figure 4(c) shows an example. It turns out that the diametric ball of a shield subfacet lies in the union of the vertex ball $\widehat{u}$ and the diametric ball of the corresponding shield subsegment. Since $\widehat{u}$ is kept empty throughout the algorithm, it is sufficient to keep the diametric balls of shield subsegments empty to ensure that shield subfacets appear in Del $\mathcal{V}$. 


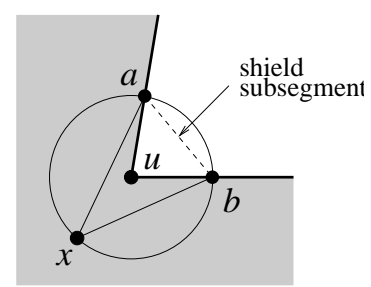

(a)

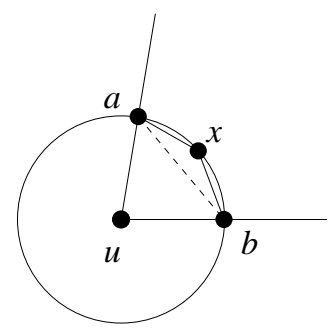

(b)

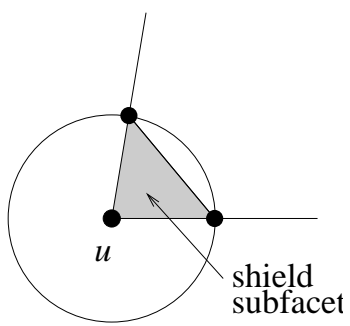

$(c)$

Figure 4: Shield subsegment, shield subfacet, and splitting.

In INITIALIZE, we only insert the points where the incident edges of $u$ intersects the boundary of $\widehat{u}$, and split shield subsegments that correspond to sectors with angles $\pi$ or more. The encroachment of shield subsegments is handled in the next phase Conform. The following technical lemma will be useful.

Lemma 3.1 Let $u$ be a vertex. Let $B$ be the ball centered at $u$ with radius $f(u) / 2$. Then

(i) The diametric ball of any shield subsegment inside $\widehat{u}$ lies in $B$.

(ii) $B$ is disjoint from any vertex ball other than $\widehat{u}$.

(iii) $B$ is disjoint from any edge or facet of $\mathcal{P}$ not incident to $u$.

Proof. The diametric ball of any shield subsegment inside $\widehat{u}$ has radius at most $f(u) / 4$. So any point in it is at distance $f(u) / 2$ or less from $u$. This proves (i). For any vertex ball $\widehat{w} \neq \widehat{u}$, its radius is equal to $f(w) / 4 \leq\|u-w\| / 4$. Also, $\operatorname{radius}(B)=f(u) / 2 \leq\|u-w\| / 2$. So the distance from $B$ to $\widehat{w}$ is at least $\|u-w\| / 4$. This proves (ii). The correctness of (iii) follows from the fact that the distance between $u$ and an non-incident edge or facet is at least $f(u)$.

\subsection{Edge splitting}

Edges are split in both the CONFORM and ReFine phases using a subroutine SplitEdges, which recovers the edges of $\mathcal{P}$ as union of Delaunay edges. SplitEdges splits any subsegment (sharp, non-sharp or shield) that is encroached using SplitE until no such segment exists.

\section{$\operatorname{SplitE}(e)$}

If $e$ is a shield subsegment, split it with SOS else insert the midpoint of $e$ in $\mathcal{V}$ and update $\operatorname{Del} \mathcal{V}$.

Notice that any point inserted by SPLITE cannot encroach the vertex balls of sharp vertices. When SplitEdges terminates, each edge of $\mathcal{P}$ appears as a union of Delaunay edges.

\subsection{Facet splitting}

After we recover all the edges, we start splitting facets using a subroutine SPLiTFACETs so that they appear in Del $\mathcal{V}$ as union of subfacets. Standard Delaunay refinement insists that 
no encroached subfacet exists. While such a condition can be enforced for subfacets on the boundary $\mathcal{B}$ of the bounding box, it may never be satisfied for all other subfacets due to the presence of small angles. Therefore, for all subfacets other than the ones on $\mathcal{B}$, we check only that the subfacets appear in Del $\mathcal{V}$. We argue that such a condition can be satisfied for a polyhedron after sufficient but finite amount of splitting.

Let $h$ be a subfacet that is to be split. We basically insert the circumcenter of $h$ if it does not encroach a subsegment. The procedure SPLITF below gives the details.

$\operatorname{SpLiTF}(h)$

(i) Compute the circumcenter $c$ of $h$;

(ii) Let $F$ be the facet containing $h$. If $c$ does not encroach any subsegment, insert $c$ into $\mathrm{V}$ and update Del $\mathcal{V}$. Otherwise, reject $c$ and

(a) pick a subsegment $g$ encroached by $c$ with preference for those in $\partial F$ or on $F$ (shield subsegment), and

(b) call $\operatorname{SpLitE}(g)$.

\subsection{Ball splitting}

In the CONFORM phase, after all the edges and facets are recovered, further splittings are done using the subroutine SplitBalls to reduce the diametric balls of the sharp subsegments roughly to the order of local feature sizes. In order to avoid the computation of local feature sizes, this is achieved in a roundabout way. SplitBalls splits any subfacet or subsegment $h$ that is encroached by the midpoint of a sharp subsegment $s$ provided that $h$ and $s$ are contained in disjoint elements of $\mathcal{P}$. The intuition is that some of the new vertices inserted to split these subfacets or subsegments will encroach $s$ and cause $s$ to split. We claim (Proof of Theorem 4.1) that, at the end of SplitBalls, all diametric balls of sharp subsegments become small.

At the end of the CONFORM phase, for each sharp subsegment, we double the radius of its diametric ball with the center fixed and call this a protecting ball. These protecting balls and the vertex balls at the sharp vertices constitute the entire set of protecting balls for the REFINE phase. Although sharp subsegments may be subdivided further in the REFINE phase, the protecting balls always refer to those computed at the end of the CONFORM phase.

\subsection{Refinement of tetrahedra}

As in usual Delaunay refinement we attempt to insert the circumcenters of the skinny tetrahedra in this phase. But, some modifications are needed to handle small angles. We disallow the insertion of the circumcenters of skinny tetrahedra inside any protecting ball. The reason is that once these points are allowed to be inserted, they can cause perpetual splittings of the subsegments or subfacets. The tetrahedra incident to the vertices and edges of $\mathcal{P}$ sustaining small enough angles can never satisfy the radius-edge ratio condition. So, at some stage of the algorithm, these tetrahedra should not be split. This means that some skinny tetrahedra may remain at the end. We prove that all such tetrahedra lie close to sharp vertices or edges. 


\subsection{QMESH}

The following algorithm QMesH triangulates $\mathcal{P}$ with the claimed theoretical guarantees. QMESH uses the following subroutine ENCROACH to test whether a point $c$ can be inserted and if not, return the appropriate point to be inserted.

\section{$\operatorname{ENCROACH}(c)$}

1. If $c$ does not encroach any subsegment or subfacet, return $c$.

2. If $c$ encroaches some subsegment $e$, reject $c$ and if $e$ is a shield subsegment, return the point given by SOS; else return the midpoint of $e$.

3. If $c$ encroaches some subfacet $h$, reject $c$ and if the circumcenter $p$ of $h$ does not encroach any subsegment, return $p$; else reject $p$ and return the point as in case 2 .

\section{$\mathrm{QMesh}(\mathcal{P})$}

Initialize. Initialize $\mathcal{V}$ to be the set of vertices of $\mathcal{P}$. Compute the vertex balls. Insert the intersections between their boundaries and the edges of $\mathcal{P}$ into $\mathcal{V}$. If any shield subsegment forms a sector with angle $\pi$ or more, split it with SOS. Compute Del $\mathcal{V}$.

CONFORM. Repeatedly apply a rule from the following list until no rule is applicable. Rule $i$ is applied if it is applicable and no rule $j$ with $j<i$ is applicable.

Rule 1(SplitEdGES). If there is an encroached subsegment $e$, call $\operatorname{SplitE}(e)$. Rule 2(SplitFacets). If there is a subfacet $h \subset \mathcal{B}$ that is encroached, or if $h \not \subset \mathcal{B}$ and $h$ does not appear in $\operatorname{Del} \mathcal{V}$, call $\operatorname{SpLiTF}(h)$.

Rule 3(SplitBalls). Let $s$ be a sharp subsegment. If the midpoint of $s$ encroaches a subsegment or subfacet $h$, where $h$ and $s$ are contained in disjoint elements of $\mathcal{P}$, split $h$ accordingly using $\operatorname{SplitE}(h)$ or $\operatorname{SpLiTF}(h)$.

At the end of Conform, we double the sizes of the diametric balls of sharp subsegments. These expanded balls and the vertex balls are the protecting balls. The sharp subsegments may be split further in the next phase, but the locations and sizes of these protecting balls do not change.

REFINE. Repeatedly apply a rule from the following list until no rule is applicable. Rule $i$ is applied if it is applicable and no rule $j$ with $j<i$ is applicable. The parameter $\rho_{0}>2 \sqrt{2} /(1-\tan (\pi / 8))$ is a constant chosen a priori.

Rule 4 (SplitEdGES). If there is an encroached subsegment $e$, call $\operatorname{SplitE}(e)$. Rule 5 (SPlitFACETS). If there is a subfacet $h \subset \mathcal{B}$ that is encroached, or if $h \not \subset \mathcal{B}$ and $h$ does not appear in $\operatorname{Del} \mathcal{V}$, call $\operatorname{SpLiTF}(h)$.

Rule 6 (SplitTet). Assume that there is a tetrahedron with radius-edge ratio exceeding $\rho_{0}$. Let $z$ be its circumcenter. If $z$ does not lie on or inside any protecting ball, then

- compute $p:=\operatorname{ENCROACH}(z)$, and 
- insert $p$ into $\mathcal{V}$ and update $\operatorname{Del} \mathcal{V}$.

QMesh can be modified to improve the threshold for $\rho_{0}$ to $2+\epsilon$ for any fixed $\epsilon>0$. See the remark at the end of Section 4.3.3. This will worsen the output angles at the sharp vertices though. Our implementation is based on the above description and we find that the threshold of 2.2 for $\rho_{0}$ can be used in the experiments without any problem.

We left out the details of the implementation of steps such as checking whether the circumcenters of skinny tetrahedra lie inside a protecting ball. There is a lot of room for experimentation and variations. We offer some suggestions below that make the computation local.

To check whether the circumcenter $p$ of a skinny tetrahedron lies inside a protecting ball, first find the tetrahedron $\tau$ that contains $p$ by a walk. If the protecting balls that intersect $\tau$ are recorded at $\tau$, one can easily determine the protecting balls containing $p$. The initialization of such information can be done at the end of CONFORM by walking from sharp subsegments to all tetrahedra intersected by protecting balls. Afterwards, with each update of the Delaunay triangulation, the information can be updated with local computations only.

\section{Analysis}

In this section, we prove that QMesh terminates with a graded Delaunay mesh. All tetrahedra have bounded radius-edge ratio, except possibly those near sharp vertices and edges (in terms of local feature sizes). An important aspect of QMESH is that, sometimes it attempts to insert a point, but eventually rejects it. These rejected points are as crucial as the inserted ones in the analysis.

We have seen that the original polyhedron to be meshed is enclosed with a bounding box whose boundary is $\mathcal{B}$. The main purpose of this box is to disallow any point to be inserted outside the box so that one can claim termination by applying a packing argument within a bounded domain. The set of points, say $P$, that are inserted or rejected while conforming to $\mathcal{B}$ maintain a lower bound on their distances to all other existing points by standard arguments of encroachment $[3,17]$. In the analysis we skip explicit arguments about $P$ and focus on the set of points, say $Q$, that are inserted or rejected for conforming to the edges and facets of the original polyhedron. Of course, we do not lose any generality by doing so as the lower bounds on distances for $P$ are dominated by those for $Q$.

In sections 4.1 and 4.2 , we analyze some critical distances when subsegments and subfacets are considered for splitting. These results are instrumental to proving that rules 1,2 , 4, and 5 never insert any vertex too close to existing ones. Then, in sections $4.3 .1,4.3 .2$, and 4.3.3 we prove lower bounds on the distances among the vertices created in Conform and REFINE. These lower bounds allow us to prove the termination and the mesh quality in Section 4.3.4. Recall that $\alpha_{m}>0$ denotes the smallest input angle in $\mathcal{P}$.

First of all, we show that the vertex balls of sharp vertices are kept empty.

Lemma 4.1 Throughout QMESH, no vertex is inserted inside the vertex ball of any sharp vertex.

Proof. Only the splitting of a subfacet may possibly insert a vertex inside a vertex ball $\widehat{u}$. 
In this case, the vertex inserted is the circumcenter $x$ of a subfacet $h$. We show that this is impossible by deriving a contradiction.

Let $F$ be the facet containing $h$. Since no subsegment is encroached when $h$ is split, $x$ lies in the interior of $F$. The facet $F$ is incident to $u$; otherwise, $F$ is disjoint from $\widehat{u}$ by Lemma 3.1(iii), contradicting the fact that $x \in F$ lies inside $\widehat{u}$.

Let $x$ lie inside a sector on $\widehat{u} \cap F$ bounded by $a u$ and $b u$, where $a b$ is a shield subsegment. If $x$ lies inside the shield subfacet $a b u$, a vertex of $h$ would have encroached $a b$, contradicting the fact that no subsegment is encroached. If $x$ does not lie inside $a b u, x$ would have been rejected for encroaching $a b$, contradicting the fact that $x$ is inserted.

\subsection{Critical distances: subsegments}

In this section, we study the effects of splitting an encroached subsegment in some situations. Our goal is to show that the distances from the inserted vertex $x$ to other vertices of $\mathcal{P}$ are $\Omega(f(x))$. In Lemma 4.2, we first tackle the case of splitting a shield subsegment, and we study encroachment due to points on the edges of $\mathcal{P}$. We extend this result in Lemma 4.3 to all subsegments, shield and non-shield.

Lemma 4.2 Let $a b$ be a shield subsegment that lies on a facet $F$ of $\mathcal{P}$. Assume that ab is encroached by either an endpoint of a subsegment or the midpoint of a sharp subsegment, which is different from $a b$. Let $x$ be the vertex to be inserted to split ab. There is a constant $\lambda_{1}>0$ such that:

(i) For any element $E$ of $\mathcal{P}$ such that $E \neq F$ and $E \nsubseteq \partial F$, the distance between $x$ and $E$ is at least $\lambda_{1} f(x)$.

(ii) For any vertex $v \in \mathcal{V},\|v-x\| \geq \lambda_{1} f(x)$.

Proof. We prove the lemma for the constant $\lambda_{1}=\sin ^{2} \alpha_{m} \sin (\pi / 4) / 10$. Let $u$ be the sharp vertex whose vertex ball contains $a b$. By the Lipschitz condition, $f(x) \leq f(u)+\|u-x\|$. Also, $\|u-x\|=f(u) / 4$. This gives

$$
f(u) \geq \frac{4}{5} f(x) .
$$

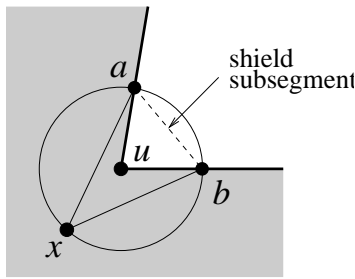

(a)

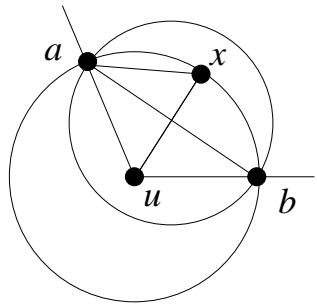

(b)

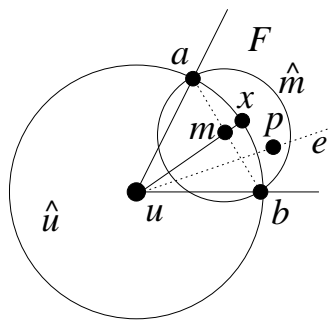

(c)

Figure 5: Splitting a shield subsegment.

We first bound $\|a-x\|$ from below in the case where the sector $a u b$ on $F$ has angle less than $\pi$ and $a b$ is not encroached by $u$. See Figure 5(c). Let $p$ be the point encroaching $a b$ (subsegment 
endpoint or midpoint of a sharp subsegment). Let $m$ be the midpoint of $a b$ and let $\widehat{m}$ be the diametric ball of $a b$. Notice that the sector $a u b$ lies on the sector $\widehat{u} \cap F$. By Lemma 3.1(iii), $\widehat{m}$ cannot intersect the edges in $\partial F$ other than the two edges incident to $u$, which bound $\widehat{u} \cap F$. By Lemma 3.1(i) and (iii), $p$ must lie on some input edge $e$ incident to $u$. Also, $p$ lies outside $\widehat{u}$ : if $p$ is a subsegment endpoint, $p$ does not lie inside $\widehat{u}$ by Lemma 4.1 ; otherwise, $p$ is the midpoint of sharp subsegment and no sharp subsegment lies inside a vertex ball by definition. We claim that $e$ is not a boundary edge of $F$. Suppose the contrary. Then $p$ lies on $e$ which bounds the sector $\widehat{u} \cap F$, and $p$ lies outside $\widehat{u}$. But $\widehat{m}$ does not intersect the bounding edges of the sector $\widehat{u} \cap F$ outside $\widehat{u}$. So $p$ cannot lie on or inside $\widehat{m}$, a contradiction. This proves our claim that $e$ is not a boundary edge of $F$. Also, it cannot be coplanar with $F$. Otherwise, for $p \in e$ to encroach $\widehat{m}, e$ would have to cut through the sector aub which is impossible. It follows that the angle between $e$ and $F$ is an input angle which is at least $\alpha_{m}$. Since $p$ lies on or inside $\widehat{m},\|a-m\| \geq\|p-m\|$. Since $p$ does not lie inside $\widehat{u},\|p-u\| \geq f(u) / 4$. Thus the distance between $p$ and $F$ is at least $f(u) \sin \alpha_{m} / 4$, which implies that $\|p-m\| \geq f(u) \sin \alpha_{m} / 4$. Hence

$$
\|a-x\| \geq\|a-m\| \geq \frac{\sin \alpha_{m}}{4} f(u) \stackrel{(4.1)}{\geq} \frac{\sin \alpha_{m}}{5} f(x) .
$$

This finishes the proof of the lower bound of $\|a-x\|$ in the case of Figure 5(c).

We proceed to prove (i). Let $E$ be an element of $\mathcal{P}$ such that $E \neq F$ and $E \nsubseteq \partial F$. There are two cases.

Case 1: $E$ is disjoint from $F$. Then the distance between $E$ and $x$ is at least $f(x)$.

Case 2: $E$ is adjacent to $F$. We claim that $x$ is not further from $a u$ and $b u$ than all the edges of $F$. First, by Lemma 3.1(iii), we only need to prove the claim for the bounding edges of the sector $\widehat{u} \cap F$. Since the sector $a u b$ lies on the sector $\widehat{u} \cap F$, the shortest line segment connecting $x$ to the bounding edges of $\widehat{u} \cap F$ must link to $u$ or intersect $a u$ or $b u$. This proves our claim.

We show that the distance $D$ between $x$ and $a u$ is at least $f(x) \sin \alpha_{m} \sin (\pi / 4) / 5$. Refer to Figure 5. In the case of Figure 5(a), the angle of the sector aub is $\pi$ or more, so $D \geq\|u-x\|=f(u) / 4$ which is at least $f(x) / 5$ by (4.1). In the cases of Figures $5(\mathrm{~b})$ and (c), the angle of the sector $a u b$ is less than $\pi$. So the nearest point on $a u$ to $x$ lies in the interior of $a u$. In the case of Figure $5(\mathrm{~b}), u$ lies on or inside $\widehat{m}$, so $\angle a u x \geq \angle a u b / 2 \geq \pi / 4$. Thus $D \geq\|u-x\| \sin (\pi / 4)=f(u) \sin (\pi / 4) / 4$ which is at least $f(x) \sin (\pi / 4) / 5$ by (4.1). In the case of Figure 5(c), $u$ does not lie inside $\widehat{m}$, so $\angle x a u \geq 3 \pi / 8>\pi / 4$. Thus $D \geq\|a-x\| \sin (\pi / 4)$ which is at least $f(x) \sin \alpha_{m} \sin (\pi / 4) / 5$ by (4.2).

If $E$ and $F$ are coplanar, the distance between $x$ and $E$ is at least $D>\lambda_{1} f(x)$. If $E$ and $F$ are not coplanar, the angle of any wedge induced by $E$ and $F$ is at least $\alpha_{m}$. It follows that the distance between $x$ and $E$ is at least $D \sin \alpha_{m}>\lambda_{1} f(x)$.

Next, we prove (ii). There are two cases.

Case 1: the sector $a u b$ has angle $\pi$ or more, or $a b$ is encroached by $u$. We have only executed InItialize and possibly Rule 1 in CONFORM so far. Thus all vertices in $\mathcal{V}$ lie on input edges or the intersections between boundaries of vertex balls and facets. By Lemma 3.1(iii), the vertex nearest $x$ is either $u$ or a vertex on an incident edge $e$ of $u$. In the first case, by (4.1), $\|u-x\|=f(u) / 4 \geq f(x) / 5>\lambda_{1} f(x)$. Consider the second case. 
If $e$ is not a boundary edge of $F$, the distance between $x$ and $e$ is at least $\lambda_{1} f(x)$ by (i). If $e$ is a boundary edge of $F$, we have shown in proving (i) that the distance from $x$ to $\partial F$ is at least the distance from $x$ to $a u$, which is at least $f(x) \sin \alpha_{m} \sin (\pi / 4) / 5>\lambda_{1} f(x)$.

Case 2: $a b$ is not encroached by $u$. Then $\angle a u b \leq \pi / 2$. Any vertex encroaching $\widehat{m}$ must lie on an element of $\mathcal{P}$; otherwise, such a vertex would have been rejected by QMESH. It follows that any vertex in $\mathcal{V} \backslash F$ are further away than the lower bound on distances in (i). Consider the vertices in $\mathcal{V} \cap F$. We claim that $\widehat{m} \cap F$ does not contain any shield subsegment endpoint other than $a$ and $b$. Clearly, all shield subsegments inside $\widehat{u} \cap F$ other than $a b$ lie outside $\widehat{m}$. Since the shield subsegments outside $\widehat{u}$ lie inside other vertex balls, they are disjoint from $\widehat{m}$ by Lemma 3.1(i) and (ii). This proves our claim. Since $\widehat{m} \cap F$ does not contain any shield subsegment endpoint other than $a$ and $b, \widehat{m}$ does not contain any vertex in the interior of $F$ because such a vertex would have been rejected by QMesh. Thus the distance from $x$ to any vertex in $\mathcal{V} \cap F$ is at least the distance from $x$ to the boundary of $\widehat{m}$. This distance is equal to $\|a-x\|(\sin (\angle a x u)-\cos (\angle a x u)) \geq\|a-x\|(\sin (3 \pi / 8)-\cos (3 \pi / 8))>\|a-x\| / 2$. By (4.2), we conclude that the distance from $x$ to any vertex in $\mathcal{V} \cap F$ is greater than $\lambda_{1} f(x)$.

Next, we extend the lower bound on the inter-vertex distance in Lemma 4.2 to all subsegments, shield and non-shield.

Lemma 4.3 Let $x$ be the vertex that splits a subsegment ab. Let $p$ be an endpoint of a subsegment or the midpoint of a sharp subsegment, which is different from ab. Suppose that $p$ encroaches ab. Then for all $v \in \mathcal{V},\|v-x\|=\Omega(f(x))$.

Proof. If $a b$ is a shield subsegment, Lemma 4.2(ii) implies that $\|v-x\|=\Omega(f(x))$ for all $v \in \mathcal{V}$. Suppose that $a b$ is a non-shield subsegment. Let $e$ be the edge containing $a b$. We first show that $\|p-x\|=\Omega(f(x))$. There are two cases.

Case 1: $p$ lies on an edge $e^{\prime}$ of $\mathcal{P}$ (including its endpoints). Then $e \neq e^{\prime}$ in order that $p$ encroaches $a b$. If $e$ and $e^{\prime}$ are disjoint, then $\|p-x\| \geq f(x)$. If $e$ and $e^{\prime}$ are adjacent, they must make an acute angle in order that $p$ encroaches $a b$. Let $u$ be the sharp vertex $e \cap e^{\prime}$. As $x$ lies outside $\widehat{u},\|u-x\| \geq f(u) / 4$. The Lipschitz condition implies that $f(x) \leq f(u)+\|u-x\| \leq 5\|u-x\|$. Since the angle between $e$ and $e^{\prime}$ is at least $\alpha_{m}$, $\|p-x\| \geq\|u-x\| \sin \alpha_{m} \geq f(x) \sin \alpha_{m} / 5$.

Case 2: $p$ lies in the interior of a facet $F$ of $\mathcal{P}$. So $p$ was inserted on the boundary of a vertex ball $\widehat{w}$ to split a shield subsegment on $F$. In order that $p$ encroaches $a b, w$ cannot be an endpoint of $e$, which implies that both $f(x)$ and $f(w)$ are at most $\|w-x\|$. Thus $\|p-x\|$ is at least the distance from $x$ to $\widehat{w}$ which is $\|w-x\|-f(w) / 4 \geq 3\|w-x\| / 4 \geq 3 f(x) / 4$.

Since $p$ encroaches $a b$, the above analysis implies that the radius of the diametric ball of $a b$ is $\Omega(f(x))$. Let $v$ be any vertex in $\mathcal{V}$. If $v$ does not lie inside the diametric ball of $a b$, then clearly $\|v-x\|=\Omega(f(x))$. Otherwise, $v$ encroaches $a b$. It follows that $v$ must lie on an element of $\mathcal{P}$; otherwise, $v$ would be the circumcenter of some skinny tetrahedron, and QMESH would have rejected $v$ for encroaching $a b$. Similarly, $v$ was not inserted by QMESH to split a subfacet as 
$v$ would be rejected for encroaching $a b$. So $v$ was inserted on an edge or to split a shield subsegment. We can apply the same analysis in cases 1 and 2 to show that $\|v-x\|=\Omega(f(x))$.

\subsection{Critical distances: subfacets}

Our goal is to show that when a non-Delaunay subfacet $h$ splits, the distances from its circumcenter $x$ to the existing vertices and the elements of $\mathcal{P}$ are $\Omega(f(x))$. The major challenge is to show that the circumradius of $h$ is $\Omega(f(x))$. Due to our new strategy of splitting non-Delaunay subfacets, the analysis for the standard Delaunay refinement does not apply.

In this section, we focus on the case where the circumballs of $h$ contain vertices on the elements of $\mathcal{P}$ only, and defer the full analysis until Section 4.3. We first look at some important special cases in Section 4.2.1 and prove a lower bound on the distance from $x$ to any encroaching point. In Section 4.2.2, we apply these results to bound the circumradius of $h$ from below. The lower bounds on the distances from $x$ to the existing vertices and the elements of $\mathcal{P}$ then follow. The next lemma on the distance between $x$ and sharp vertices will be useful.

Lemma 4.4 Let $x$ be the circumcenter of a subfacet on a facet $F$. For any sharp vertex $u$ of $F,\|u-x\| \geq f(x) / 9$.

Proof. Let $h$ be the subfacet with the circumcenter $x$. Let $C$ be the circumcircle of $h$. We claim that for any sharp vertex $u$ of $F,\|u-x\| \geq f(u) / 8$. Otherwise, since $u$ does not lie inside $C, \operatorname{radius}(C) \leq\|u-x\|<f(u) / 8$. Thus the vertices of $h$ lie inside the vertex ball $\widehat{u}$, but this contradicts Lemma 4.1. By the claim, the Lipschitz condition implies that $f(x) \leq f(u)+\|u-x\|<9\|u-x\|$.

\subsubsection{Splitting subfacets: special cases}

The next three lemmas show that the distance between the circumcenter $x$ of a subfacet from an encroaching point is $\Omega(f(x))$ under some special conditions. These special cases will arise in the analysis in Section 4.2.2 and Section 4.3.

Lemma 4.5 Assume that no subsegment is encroached by a vertex. Let $E$ and $F$ be two noncoplanar adjacent facets. Let $x$ be the circumcenter of a subfacet on $F$. Let $q$ be the closest point to $x$ on the intersection line of the support planes of $E$ and $F$. Suppose that one of the the following holds:

(i) $q x$ intersects an edge in $\partial F$ disjoint from $E$.

(ii) $q \in E$ and an edge in $\partial F$ intersects $q x$ at $x$ or in its interior.

(iii) $q$ is an isolated vertex in $E \cap F$.

Then for any point $u \in E,\|u-x\|=\Omega(f(x))$.

Proof. Consider (i) (Figure 6(a)). Let $p$ be the intersection point of $q x$ and the edge in $\partial F$. Since this edge is disjoint from $E, \max \{\|p-x\|,\|u-x\|\} \geq f(x)$. If $\|u-x\| \geq f(x)$, we are 
done. Otherwise, $\|p-x\| \geq f(x)$ and $\|q-x\| \geq\|p-x\| \geq f(x)$. If the wedge of $E$ and $F$ containing $u$ and $x$ is sharp, then $\|u-x\| \geq\|q-x\| \sin \alpha_{m} \geq f(x) \sin \alpha_{m}$. If the wedge is non-sharp, then $\angle u q x \geq \pi / 2$ and so $\|u-x\| \geq\|q-x\| \geq f(x)$.

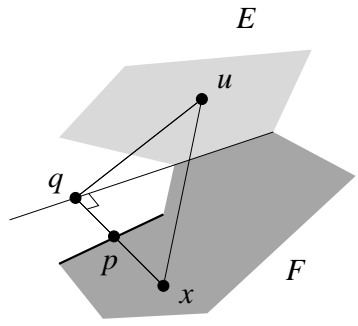

(a)

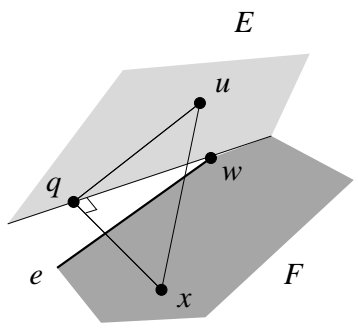

(b)

Figure 6: Illustration for Lemma 4.5 (i) and (ii).

Consider (ii) (Figure 6(b)). Let $e$ be the edge in $\partial F$ intersecting $q x$ at $x$ or in its interior. We assume that (i) does not apply, so $e$ is incident to a vertex $w$ in $E \cap F$. We show that $\|q-x\|=\Omega(f(x))$, which implies that $\|u-x\|=\Omega(f(x))$ as in case 1 . The angle between $q w$ and $e$ is acute. As $q \in E$, this angle is at least an input angle at $w$. So $w$ is sharp and this angle is at least $\alpha_{m}$. Thus $\angle x w q \geq \alpha_{m}$. By Lemma 4.4, $\|w-x\| \geq f(x) / 9$. It follows that $\|q-x\| \geq\|w-x\| \sin \alpha_{m} \geq f(x) \sin \alpha_{m} / 9$.

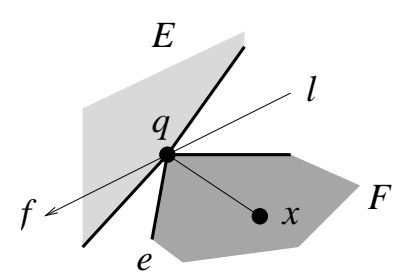

(a)

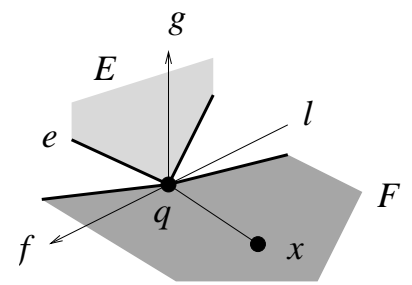

$(c)$

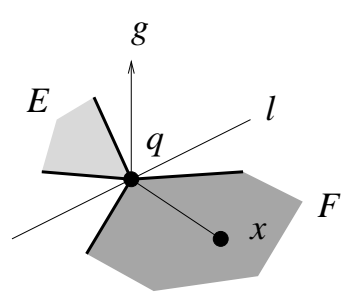

$(b)$

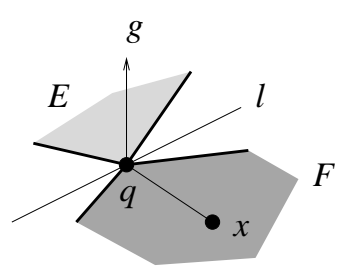

$(d)$

Figure 7: Illustration for Lemma 4.5 (iii).

Consider (iii) (Figure 7). We assume that (ii) does not apply. So no edge of $F$ intersects $q x$ at $x$ or in its interior. As $x$ is the circumcenter of a subfacet, $x$ lies in the interior of $F$. So $q x$ does not lie on an edge of $F$. Let $\ell$ be the intersection line of the support planes of $E$ and $F$. We first show that $q$ is a sharp vertex. There are two cases.

Case 1: $E$ does not lie strictly on one side of $\ell$ locally at $q$. So a halfline $f$ on $\ell$ delimited by $q$ intersects $E$ locally at $q$. As $q x \subseteq F$ and $x$ lies in the interior of $F$, the quadrant formed 
by $q x$ and $f$ on the plane of $F$ must contain an edge $e$ of $F$. See Figure 7(a). The edge $e$ makes an acute angle with $E$. So $q$ is sharp.

Case 2: $E$ lies strictly on one side of $\ell$ locally at $q$. Let $g$ be the halfline on the support plane of $E$ that is delimited by $q$ and perpendicular to $\ell$. If the two edges of $E$ incident to $q$ lie in one of the quadrants formed by $\ell$ and $g$, then they make an acute angle at $q$. See Figure 7(b). So $q$ is sharp. Suppose that the two edges of $E$ incident to $q$ lie in different quadrants formed by $\ell$ and $g$. There are two cases.

Case 2.1: $F$ does not lie strictly on one side of $\ell$ locally at $q$. A halfline $f$ on $\ell$ delimited by $q$ intersects $F$ locally at $q$. The quadrant formed by $f$ and $g$ contains an edge $e$ of $E$ incident to $q$. See Figure 7(c). Thus $e$ makes an acute angle with $F$ and $q$ is sharp.

Case 2.2: $F$ lies strictly on one side of $\ell$ locally at $q$. Then the four edges of $E$ and $F$ incident to $q$ form a convex cone with apex $q$. See Figure $7(d)$. The sum of the four face angles of the convex cone is less than $2 \pi$, which implies that at least one face angle is acute. So $q$ is sharp.

This finishes the proof of $q$ being sharp. By Lemma 4.4, $\|q-x\| \geq f(x) / 9$. If the wedge of $E$ and $F$ containing $u$ and $x$ is sharp, then $\|u-x\| \geq\|q-x\| \sin \alpha_{m} \geq f(x) \sin \alpha_{m} / 9$. If the wedge is non-sharp, then $\angle u q x \geq \pi / 2$ and so $\|u-x\| \geq\|q-x\| \geq f(x) / 9$.

Lemma 4.6 Assume that no subsegment is encroached by a vertex. Let $E$ and $F$ be two noncoplanar adjacent facets. Let $x$ be the circumcenter of a subfacet $h$ on $F$. Let $u$ be a point on $E$ such that $u$ and $x$ lie on a sharp wedge of $E$ and $F$. Let $q$ be the closest point to $x$ on the intersection line of the support planes of $E$ and $F$. Suppose that qu intersects an edge $e$ in $\partial E$. Also, suppose that $e$ is incident to a vertex $w$ in $E \cap F$ such that $\|w-x\|=\Omega(f(x))$. Then $\|u-x\|=\Omega(f(x))$.

Proof. Let $x^{\prime}$ be the orthogonal projection of $x$ onto the support plane of $E$. Since $u$ and $x$ lie on a sharp wedge of $E$ and $F, x^{\prime}$ lie on this sharp wedge too. There are two cases.

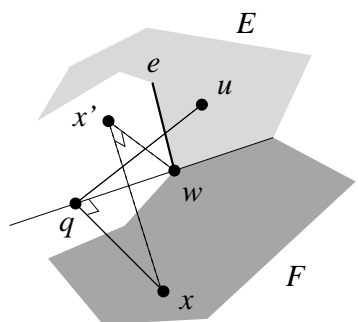

(a)

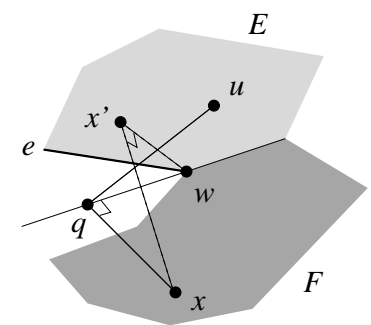

(b)

Figure 8: Illustration for Lemma 4.6.

Suppose that the angle between $e$ and $q w$ is greater than or equal to $\angle x^{\prime} w q$. See Figure 8 (a). By Lemma 2.1, $\angle u w x$ is greater than the angle between $e$ and $w x$. The angle between $e$ and $w x$ is at least an input angle which is at least $\alpha_{m}$. So $\angle u w x \geq \alpha_{m}$, which implies that $\|u-x\| \geq\|w-x\| \sin \angle u w x=\Omega(f(x))$. 
Suppose that the angle between $e$ and $q w$ is less than $\angle x^{\prime} w q$. See Figure 8(b). The angle between $e$ and $w x$ is at least $\alpha_{m}$. By Lemma 2.1, $\angle q w x$ is greater than the angle between $e$ and $w x$, which implies that $\angle q w x \geq \alpha_{m}$. Thus, $\|q-x\|=\|w-x\| \sin \angle q w x=\Omega(f(x))$. Then $\|u-x\| \geq\left\|x-x^{\prime}\right\| \geq\|q-x\| \sin \alpha_{m}=\Omega(f(x))$.

Lemma 4.7 Assume that no subsegment is encroached by a vertex. Let $E$ and $F$ be two noncoplanar adjacent facets. Let $h$ be a subfacet on $F$ with circumcenter $x$. Suppose that the diametric ball of $h$ contains a point $u$ on $E$ such that $u$ does not encroach any subsegment. Then the following hold:

(i) F contains the segment joining $x$ and the orthogonal projection of $u$ onto the support plane of $F$.

(ii) If the wedge of $E$ and $F$ containing $u$ and $x$ is non-sharp, then $\|u-x\|=\Omega(f(x))$.

(iii) Suppose that the wedge of $E$ and $F$ containing $u$ and $x$ is sharp. Let $q$ be the nearest point to $x$ on the intersection line of the support planes of $E$ and $F$. If $q \notin E \cap F$ or $q$ lies on a non-sharp edge in $E \cap F$, then $\|u-x\| \geq \delta f(x)$ for some constant $\delta>0$.

Proof. Let $B$ and $C$ be the diametric ball and circumcircle of $h$, respectively. Let $\ell$ be the intersection line of the support planes of $E$ and $F$. Let $u^{\prime}$ be the orthogonal projection of $u$ onto the support plane of $F$.

Consider (i). Note that $u^{\prime}$ lies inside $C$ as $u$ lies inside $B$. Assume to the contrary that (i) is false. So an edge $e$ in $\partial F$ separates $x$ and $u^{\prime}$ inside $C$. By the emptiness of $C$, e contains a chord $a b$ of $C$, which lies within a subsegment on $e$. See Figure $9(\mathrm{a})$. The point $u$ lies outside the diametric ball of $a b$ as $u$ does not encroach any subsegment by assumption. The bisector of $B$ and the diametric ball of $a b$ is the plane $H$ through $a b$ perpendicular to $F$. Since $a b$ separates $u^{\prime}$ and $x, H$ bounds an open halfspace $H^{-}$such that $u^{\prime} \in H^{-}$and $x \notin H^{-}$. Since $u$ does not lie inside the diametric ball of $a b$ but inside $B, u \notin H^{-}$. But this contradicts the fact that $u^{\prime}$ is the orthogonal projection of $u$.

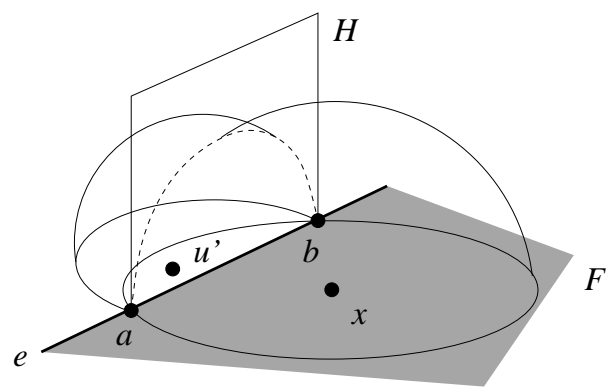

(a)

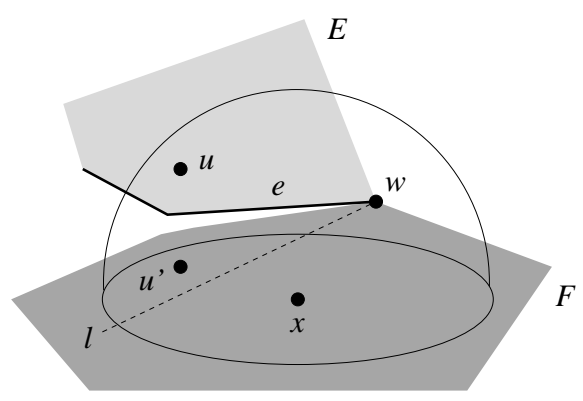

(b)

Figure 9: Illustration for Lemma 4.7 (i) and (ii).

Consider (ii). Since the wedge of $E$ and $F$ containing $u$ and $x$ is non-sharp, $u^{\prime}$ and $x$ do not lie on the same side of $\ell$. No edge in $E \cap F$ intersects $C$. Otherwise, such an edge crosses $C$ completely (due to the emptiness of $C$ ) and as this edge lies on $\ell$, it must cross $x u^{\prime}$, contradicting 
(i). Since $B$ contains $u \in E, B$ must intersect an edge $e$ in $\partial E$ above $F$ such that $e$ is closer to $x$ than $u$. See Figure 9(b). If $e$ is disjoint from $F$, then $\|u-x\| \geq f(x)$ as $e$ is closer to $x$ than $u$. If $e$ is incident to a vertex $w$ of $F$, then $w$ lies on $\ell$ and outside $C$. Observe that the angle between $e$ and $\ell$ is acute. Since $\ell$ intersects $x u^{\prime} \subseteq F$, this angle is at least an input angle at $w$. So $w$ is sharp and $\|w-x\| \geq f(x) / 9$ by Lemma 4.4. The angle between $e$ and $w x$ is at least $\alpha_{m}$. The distance between $x$ and $e$ is at least $\|w-x\| \sin \alpha_{m} \geq f(x) \sin \alpha_{m} / 9$, and so is $\|u-x\|$.

There are two cases in proving (iii).

Case 1: $q u \nsubseteq E E$. Then an edge $e$ in $\partial E$ intersects $q u$ at $u$ or in its interior. Suppose that $e$ is disjoint from $F$. Let $p=q u \cap e$. By considering the triangle $q u x$ in Figure 10(a), we have $\max \{\|u-x\|,\|q-x\|\} \geq\|p-x\| \geq f(x)$. If $\|u-x\| \geq f(x)$, we are done. Otherwise, $\|q-x\| \geq f(x)$ implies that $\|u-x\| \geq\|q-x\| \sin \alpha_{m} \geq f(x) \sin \alpha_{m}$.

Suppose that $e$ is adjacent to $F$. So $e$ is incident to a vertex $w$ in $E \cap F$. We prove that $w$ is sharp. Then by Lemma $4.4,\|w-x\| \geq f(x) / 9$ which allows us to apply Lemma 4.6 to show that $\|u-x\|=\Omega(f(x))$. Let $H$ be the plane through $w$ orthogonal to $\ell$.

If $e$ lies on $H$ or $e$ lies on the same side of $H$ as $x$, let $g$ be the halfline in the intersection of $H$ and the support plane of $F$ such that $g$ is delimited by $w$ and lies on the same side of $\ell$ as $q x$. See Figure 10(b). Lemma 2.1 implies that the angle between $e$ and $g$ is acute as it is less than the right angle between $q w$ and $g$. As $e$ lies on $H$ or $e$ lies on the same side of $H$ as $x$, the quadrant formed by $g$ and $q w$ contains the orthogonal projection of $e$ onto the support plane of $F$. Then Lemma 2.1 implies that the angle between $e$ and $w x$ is less than one of the angles that $e$ makes with $q w$ and $g$. So the angle between $e$ and $w x$ is acute. Since $x \in F$, the angle between $e$ and $w x$ is at least an input angle at $w$. So $w$ is sharp.

If $e$ and $x$ lie on opposite sides of $H$, since $q u$ intersects $e, u$ and $e$ lie on the same side of $H$. See Figure 10(c). The argument in the previous paragraph can be applied again to show that the angle between $e$ and $w u^{\prime}$ is acute. As $u^{\prime} \in F$ by (i), we conclude that $w$ is sharp.

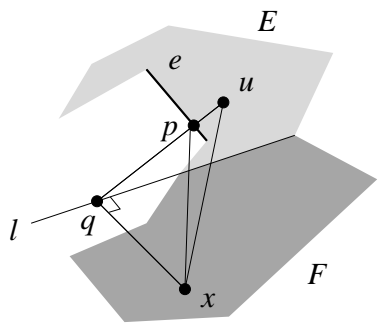

(a)

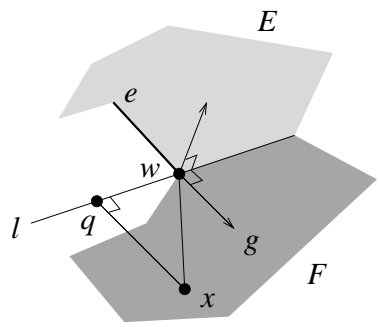

(b)

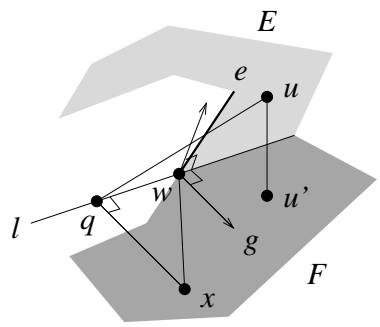

(c)

Figure 10: Illustration for Lemma 4.7 (iii).

Case 2: $q u \subseteq E$. So $q \in E$. If $q x \not \subset F$, an edge of $F$ intersects $q x$ at $x$ or in its interior. So $\|u-x\|=\Omega(f(x))$ by Lemma 4.5(ii). Suppose that $q x \subseteq F$. Then $q \in E \cap F$. The assumption of the lemma implies that $q$ lies on a non-sharp edge, say $e$, in $E \cap F$. The two conditions $q u \subseteq E$ and $q x \subseteq F$ imply that a dihedral angle of $e$ is equal to the angle of the wedge of $E$ and $F$ containing $u$ and $x$. This is a contradiction as the wedge is sharp and $e$ is non-sharp. 


\subsubsection{Splitting subfacets: general cases}

We are to show that if a subfacet $h$ is non-Delaunay and its circumballs contain vertices on the elements of $\mathcal{P}$ only, then its circumradius is $\Omega(f(x))$, where $x$ is the circumcenter of $h$. The analysis consists of three steps. The first step is a technical result (Lemma 4.8) to be used later. Second, we prove in Lemma 4.9 that $h$ has a non-empty circumball with radius $\Omega(f(x))$. Third, we prove in Lemma 4.10 that if $h$ has a large non-empty circumball, its circumradius is $\Omega(f(x))$. This further implies that the distances from $x$ to the elements of $\mathcal{P}$ are $\Omega(f(x))$.

Lemma 4.8 Assume that no subsegment is encroached by a vertex. Let $h$ be a subfacet on a facet $F$. Let $p$ be a vertex on an element $E$ of $\mathcal{P}$. If $p$ lies inside some circumball of $h$, then $E \neq F, E \nsubseteq \partial F$, and $E$ and $F$ are non-coplanar.

Proof. Assume to the contrary that $E=F$, or $E \subseteq \partial F$, or $E$ is coplanar with $F$. Then $p$ actually lies inside the circumcircle of $h$ since circumballs are finite. If $E=F$ or $E \subseteq \partial F$, this contradicts the emptiness of the circumcircle of $h$. If $E$ and $F$ are coplanar, the circumcircle of $h$ must cross a subsegment in $\partial F$ in order to contain $p$. But then $p$ encroaches this subsegment, a contradiction.

Lemma 4.9 Assume that no subsegment is encroached by a vertex. Let $x$ be the circumcenter of a subfacet $h$ such that $h \notin \operatorname{Del} \mathcal{V}$. Let $F$ be the facet of $\mathcal{P}$ containing $h$. Suppose that all vertices inside the diametric ball of $h$ lie on the elements of $\mathcal{P}$. Then there is a circumball $B$ of $h$ that contains vertices $u, v \in \mathcal{V}$ such that:

(i) $u$ lies on some element of $\mathcal{P}$.

(ii) $v$ and the center of $B$ lie in the same open halfspace bounded by the plane of $F$, and $u$ lies in the other open halfspace.

(iii) If $v$ lies on some element of $\mathcal{P}$, then $\operatorname{radius}(B) \geq \lambda_{2} f(x)-D$ where $D$ is the distance between $x$ and the center of $B$, and $\lambda_{2}>0$ is some constant.

Proof. Since $h \notin$ Del $\mathcal{V}$, the diametric ball of $h$ is non-empty. Let $H$ be the support plane of $F$. Let $H^{-}$be an open halfspace bounded by $H$ that contains some vertices inside the diametric ball of $h$. Let $H^{+}$be the opposite open halfspace.

By assumption, all vertices in $H^{-}$inside the diametric ball of $h$ lie on some elements of $\mathcal{P}$. If we move the center of the diametric ball of $h$ in the normal direction of $H$ into $H^{+}$, we obtain a system of circumballs of $h$ passing through $C$. Since $h \notin \operatorname{Del} \mathcal{V}$, one of these circumballs $B$ contain vertices on both sides of $H$, say $u \in H^{-}$and $v \in H^{+}$. It follows that (i) and (ii) are correct.

Let $E_{1}$ be a facet of $\mathcal{P}$ containing $u$ ( $u$ may lie on its boundary). Suppose that $v$ lies on an element of $\mathcal{P}$. Then $v$ lies on some facet $E_{2}$ of $\mathcal{P}$ (including the boundary of $E_{2}$ ). By Lemma 4.8 , neither $E_{1}$ nor $E_{2}$ is coplanar with $F$. 
Let $z$ be the center of $B$. If any two of $E_{1}, E_{2}$, and $F$ are disjoint, then $\operatorname{radius}(B) \geq f(z)$. The Lipschitz condition implies that $\operatorname{radius}(B) \geq f(z) \geq f(x)-D$ and we are done. The remaining possibility is that $E_{1}$ and $E_{2}$ are identical or adjacent, and both are adjacent to $F$. In the rest of the proof, it suffices to bound $\|u-x\|$ or $\|v-x\|$ from below as radius $(B) \geq$ $\max \{\|u-x\|,\|v-x\|\}-D$ by the triangle inequality. For $i=1$ and 2 , let $q_{i}$ be the closest point to $x$ on the intersection line between the support planes of $E_{i}$ and $F$. There are several cases.

If the wedge of $E_{1}$ and $F$ containing $u$ and $x$ is non-sharp. then $\|u-x\|=\Omega(f(x))$ by Lemma 4.7(ii). Suppose that the wedge of $E_{1}$ and $F$ containing $u$ and $x$ is sharp.

Case 1: $\left\|q_{i}-x\right\| \geq f(x) \sin \left(\alpha_{m} / 2\right) / 18$ for $i=1$ or 2 . Without loss of generality assume that $\left\|q_{1}-x\right\| \geq f(x) \sin \left(\alpha_{m} / 2\right) / 18$. Since the angle of the wedge of $E_{1}$ and $F$ containing $u$ and $x$ is at least $\alpha_{m},\|u-x\| \geq\left\|q_{1}-x\right\| \sin \alpha_{m}=\Omega(f(x))$.

Case 2: $\left\|q_{i}-x\right\|<f(x) \sin \left(\alpha_{m} / 2\right) / 18$ for $i=1$ and 2. If $q_{1} \notin E_{1} \cap F$ or $q_{1}$ lies on a non-sharp edge in $E_{1} \cap F$, then $\|u-x\|=\Omega(f(x))$ by Lemma 4.7 (iii). If $q_{1}$ is an isolated vertex in $E_{1} \cap F$, then $\|u-x\|=\Omega(f(x))$ by Lemma 4.5(iii). The remaining case is that $q_{1}$ lies on a sharp edge $e_{1}$ in $E_{1} \cap F$.

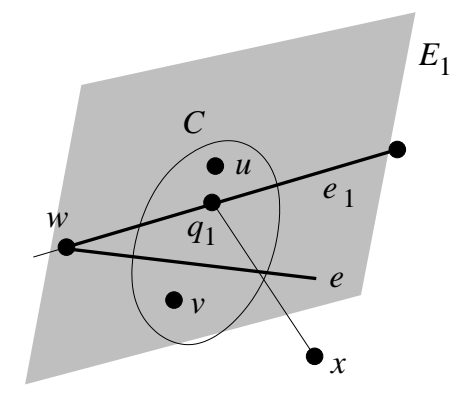

Figure 11: Case 2.1.

Case 2.1: $E_{1}=E_{2}$. In order that $B$ contains $u$ and $v$ which are on opposite sides of $F, B$ must intersect the support plane of $E_{1}$ in a circle $C$ which contains $u, v$, and $q_{1}$. By the emptiness of the circumcircle of $h, e_{1}$ crosses $C$ completely. Since $u, v \in E_{1}$, the segment $u v$ intersects $e_{1}$ as well as another edge $e$ in $\partial E_{1}$. If $e_{1}$ and $e$ are disjoint, then $\operatorname{radius}(B) \geq f(z)$. The Lipschitz condition implies that $\operatorname{radius}(B) \geq f(z) \geq f(x)-D$ and we are done. Suppose that $e_{1}$ and $e$ are adjacent. So $e_{1}$ and $e$ share a vertex $w$ in $E_{1} \cap F$. Recall that $e_{1}$ is sharp and so its endpoint $w$ is also sharp by definition. See Figure 11. Since $\left\|q_{1}-x\right\|<f(x) \sin \left(\alpha_{m} / 2\right) / 18$ by the assumption of case 2 and $\|w-x\| \geq f(x) / 9$ by Lemma 4.4 , we get $\left\|w-q_{1}\right\| \geq\|w-x\|-\left\|q_{1}-x\right\| \geq f(x) / 18$. The angle between $e_{1}$ and $e$ is at least $\alpha_{m}$. So $\left\|q_{1}-v\right\| \geq\left\|w-q_{1}\right\| \sin \alpha_{m} \geq f(x) \sin \alpha_{m} / 18$. Since the wedge of $E_{1}$ and $F$ containing $u$ and $x$ is sharp by assumption, $\angle x q_{1} v$ is non-acute. It follows that $\|v-x\| \geq\left\|q_{1}-v\right\|=\Omega(f(x))$.

Case 2.2: $E_{1} \neq E_{2}$. There are two cases depending on whether $q_{2} \in E_{2}$.

Case 2.2.1: $q_{2} \in E_{2}$. If $q_{2} \notin E_{2} \cap F$, an edge in $\partial F$ must intersect $q_{2} x$ at $x$ or its interior. So Lemma 4.5(ii) implies that $\|v-x\|=\Omega(f(x))$. Suppose that $q_{2} \in E_{2} \cap F$. If $q_{2}$ is an isolated vertex in $E_{2} \cap F$, Lemma 4.5(iii) implies that $\|v-x\|=\Omega(f(x))$. The remaining possibility is that $q_{2}$ lies on an edge $e_{2}$ in 

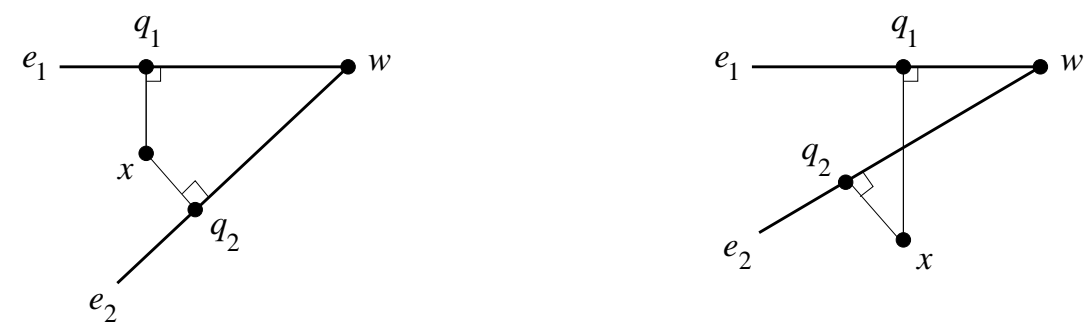

Figure 12: The possible configurations for case 2.2.1.

$E_{2} \cap F$. Observe that $e_{1} \neq e_{2}$; otherwise, $e_{1}$ would be incident to three distinct facets $E_{1}, E_{2}$, and $F$, violating the fact that $\mathcal{P}$ is a polyhedron. If $e_{1}$ and $e_{2}$ are disjoint, then $\max \left\{\left\|q_{1}-x\right\|,\left\|q_{2}-x\right\|\right\} \geq f(x)$, contradicting the assumption of case 2. If $e_{1}$ and $e_{2}$ share an endpoint, say $w$, then $w$ is sharp as it is an endpoint of the sharp edge $e_{1}$. See Figure 12. The angle between $e_{1}$ and $e_{2}$ is at least $\alpha_{m}$, and $w x$ makes an angle at least $\alpha_{m} / 2$ with $e_{1}$ or $e_{2}$. But then Lemma 4.4 implies that $\max \left\{\left\|q_{1}-x\right\|,\left\|q_{2}-x\right\|\right\} \geq\|w-x\| \sin \left(\alpha_{m} / 2\right) \geq f(x) \sin \left(\alpha_{m} / 2\right) / 9$, contradicting the assumption of case 2 .

Case 2.2.2: $q_{2} \notin E_{2}$. Then an edge $e$ in $\partial E_{2}$ intersects $q_{2} v$ at $v$ or its interior. If $e$ is disjoint from $F$, let $p=q_{2} v \cap e$. By considering the triangle $q_{2} v x$, we have $\max \left\{\|v-x\|,\left\|q_{2}-x\right\|\right\} \geq\|p-x\| \geq f(x)$, which implies that $\|v-x\| \geq f(x)$ by the assumption of case 2. Suppose that $e$ is adjacent to $F$. So $e$ is incident to a vertex $w$ in $E_{2} \cap F$. We claim that $\|w-x\| \geq f(x) / 9$. If $w$ is an endpoint of $e_{1}$, then $w$ is sharp as $e_{1}$ is sharp. So $\|w-x\| \geq f(x) / 9$ by Lemma 4.4. If $w$ is not an endpoint of $e_{1}$, then $\max \left\{\left\|q_{1}-x\right\|,\|w-x\|\right\} \geq f(x)$, which implies that $\|w-x\| \geq f(x)$ by the assumption of case 2. This proves our claim.

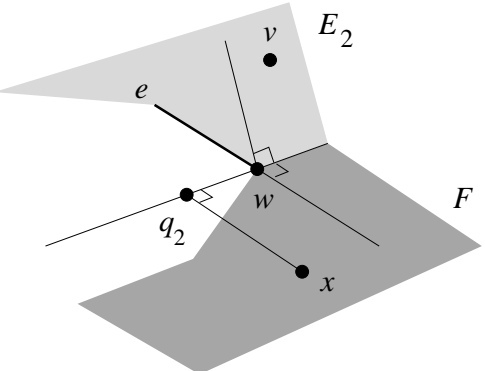

$(a)$

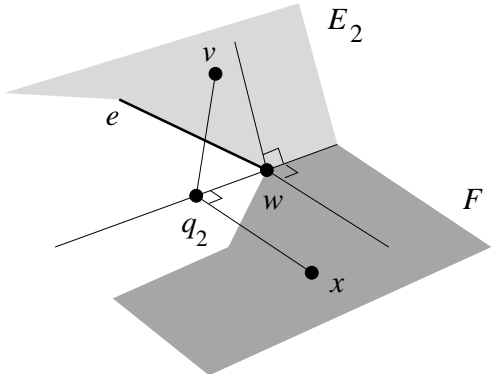

(b)

Figure 13: Case 2.2.2.

If the wedge of $E_{2}$ and $F$ containing $x$ and $v$ is sharp, we have satisfied the conditions of Lemma 4.6, which implies that $\|v-x\|=\Omega(f(x))$. Assume that the wedge is non-sharp. Let $G$ be the plane through $w$ that is orthogonal to the intersection line of the support planes of $E_{2}$ and $F$. If $x$ and $v$ do not lie on the same side of $G$, then $\angle x w v \geq \pi / 2$. See Figure 13 (a). So $\|v-x\| \geq\|w-x\| \geq$ $f(x) / 9$. If $x$ and $v$ lie on the same side of $G, e$ and $x$ also lie on the same side of $G$ as $q_{2} v$ intersects $e$. See Figure 13(b). By Lemma 2.1, $\angle x w v$ is at least the angle between $e$ and $w x$, which is at least $\alpha_{m}$. So $\|x-v\| \geq\|w-x\| \sin \alpha_{m} \geq$ $f(x) \sin \alpha_{m} / 9$. 
Based on the large circumball guaranteed by the previous lemma, we go on to show that the circumradius of the non-Delaunay subfacet is also large. This also yields a lower bound on the distances from the circumcenter to the elements of $\mathcal{P}$.

Lemma 4.10 Assume that no subsegment is encroached by a vertex. Let $x$ be the circumcenter of a subfacet $h$ on a facet $F$ of $\mathcal{P}$. Suppose that there is a circumball $B$ of $h$ centered at $z$ and $B$ contains a vertex $u$ such that:

- $u$ lies on some element of $\mathcal{P}$,

- $u$ and $z$ lie in the different open halfspaces bounded by the plane of $F$, and

- $\operatorname{radius}(B) \geq c f(x)-\|x-z\|$ for some constant $c$.

Let $k_{1}(c)=\min \left\{\frac{c \tan \alpha_{m}}{2+\tan \alpha_{m}}, \delta\right\}$ and let $k_{2}(c)=\frac{\sin \alpha_{m}}{\sqrt{2}} k_{1}(c)$, where $\delta=\Theta\left(\sin \alpha_{m}\right)$ is the constant in Lemma 4.7(iii). Then

(i) The circumradius of $h$ is at least $k_{1}(c) f(x)$.

(ii) Let $E$ be an element of $\mathcal{P}$ such that $E \neq F$ and $E \nsubseteq \partial F$. If $x$ does not encroach upon any subsegment in $\partial F$, the distance between $x$ and $E$ is at least $k_{2}(c) f(x)$.

Proof. Let $C$ be the circumcircle of $h$. Let $E_{1}$ be the element of $\mathcal{P}$ containing $u$. By Lemma 4.8, $E_{1}$ is not coplanar with $F$. Without loss of generality, assume that $F$ is horizontal and $u$ is above $F$. Let $H$ be the support plane of $F$. Let $D=\|x-z\|$. Let $u^{\prime}$ be the orthogonal projection of $u$ onto $H$. Note that $u^{\prime}$ lies inside $C$ as $u$ lies inside $B$, and $u$ and $z$ are in the different halfspaces bounded by $H$. We first prove (i).

Case 1: $E_{1}$ is an edge. If $E_{1}$ and $F$ are disjoint, then $\|u-x\| \geq f(x)$. Since $u$ and $z$ lie on opposite sides of $F, \operatorname{radius}(C) \geq\|u-x\| \geq f(x)$ and we are done. If $E_{1}$ and $F$ are adjacent, $E_{1} \cap F$ is a vertex $w$. Let $L$ be the plane through $u, u^{\prime}$, and $w$. Refer to Figure 14 which shows the cross-section on $L$.

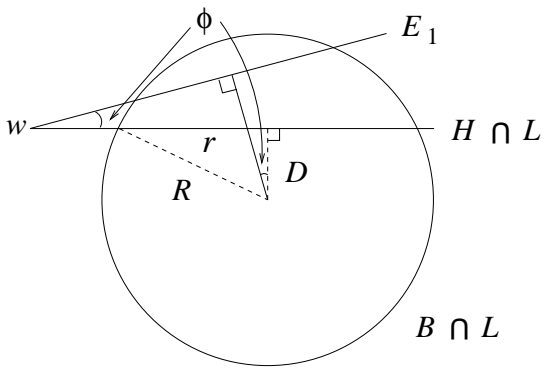

Figure 14: Cross-section on $L$.

Since $w$ lies outside $C, w$ lies outside $B \cap L$. We have

$$
\tan \phi \leq \frac{r}{D} \leq \frac{\operatorname{radius}(C)}{D}
$$


By the triangle inequality, $\operatorname{radius}(C) \geq \operatorname{radius}(B)-D$. Since $\operatorname{radius}(B) \geq c f(x)-D$ by assumption, we have

$$
\begin{aligned}
\operatorname{radius}(C) & \geq c f(x)-2 D \\
& \geq c f(x)-\frac{2 \operatorname{radius}(C)}{\tan \phi},
\end{aligned}
$$

which implies that $\operatorname{radius}(C) \geq \frac{c \tan \phi}{2+\tan \phi} f(x)$. By Lemma 4.7(i), $u^{\prime} \in F$, so $\phi \geq \alpha_{m}$ as it is at least an input angle at $w$. It follows that radius $(C) \geq \frac{c \tan \alpha_{m}}{2+\tan \alpha_{m}} f(x) \geq k_{1}(c) f(x)$.

Case 2: $E_{1}$ is a facet. If $E_{1}$ and $F$ are disjoint, then $\|u-x\| \geq f(x)$. Since $u$ and $z$ lie on opposite sides of $F, \operatorname{radius}(C) \geq\|u-x\| \geq f(x)$ and we are done. Assume that $E_{1}$ and $F$ are adjacent. The angle of the wedge of $E_{1}$ and $F$ containing $u$ and $x$ is at least $\alpha_{m}$. Let $\ell$ be the intersection line of the support planes of $E_{1}$ and $F$. Let $q^{\prime}$ be the nearest point to $u^{\prime}$ on $\ell$. There are two cases depending on whether $q^{\prime}$ is inside or outside $C$.

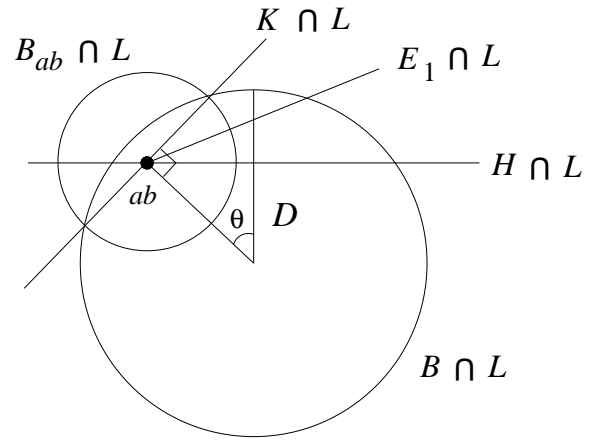

Figure 15: Cross-section on $L$.

Case 2.1: $q^{\prime}$ lies outside $C$. Let $L$ be the plane through $q^{\prime}, u$, and $u^{\prime}$. Then we can apply the analysis in case 1 to the cross-section on $L$ to conclude that $\operatorname{radius}(C) \geq$ $k_{1}(c) f(x)$.

Case 2.2: $q^{\prime}$ lies inside $C$. There are two cases.

Case 2.2.1: $E_{1} \cap F$ contains an edge $e$ that stabs $C$. The emptiness of $C$ implies that $e$ contains a chord $a b$ of $C$, and $a b$ is part of a subsegment. It also follows that $q^{\prime} \in a b$. Let $B_{a b}$ be the diametric ball of $a b$. Let $K$ be the plane that passes through $\partial B_{a b} \cap \partial B$. Let $L$ be the plane through $q^{\prime}$, $u$, and $u^{\prime}$. Consider the cross-section on $L$. Refer to Figure 15. Observe that $a b$ lies on $K$. Since no subsegment is encroached, $u$ lies outside $B_{a b}$. As $u$ lies inside $B$ by assumption, $u$ must lie on the same side of $K$ as $z$ does. This fact with the condition that $u \in E_{1}$ implies that the angle of the wedge of $E_{1}$ and $F$ containing $u$ and $x$ is at most the acute angle between $K$ and $H$, which is the angle $\theta$ in Figure 15. We have $\tan \theta \leq \frac{\operatorname{radius}(C)}{D}$. The triangle inequality implies that $\operatorname{radius}(C) \geq \operatorname{radius}(B)-D$, which is at least $c f(x)-2 D$ by assumption. Thus the same analysis in case 1 yields $\operatorname{radius}(C) \geq k_{1}(c) f(x)$.

Case 2.2.2: No edge in $E_{1} \cap F$ stabs $C$. Then the closest point to $x$ on $\ell$ lies outside $E_{1} \cap F$. Lemma 4.7 (iii) implies that $\|u-x\| \geq \delta f(x)$. Since $u$ and $z$ lie on opposite sides of $F$, radius $(C) \geq\|u-x\| \geq \delta f(x)$. 
Consider (ii). If $E$ and $F$ are disjoint, the distance between $x$ and $E$ is at least $f(x)$ and we are done. Suppose that $E$ and $F$ are adjacent. First, we claim that the distance between $x$ and $\partial F$ is at least $\operatorname{radius}(C) / \sqrt{2}$. If $C$ does not intersect $\partial F$, the claim is trivially true. Otherwise, take any subsegment $s \in \partial F$ that intersects $C$ (so $s$ crosses $C$ completely). Since $x$ does not encroach $s$ by assumption, the distance between $x$ and $s$ is at least $\operatorname{radius}(C) / \sqrt{2}$. By our claim, the distance between $x$ and $E \cap F$ is at least $\operatorname{radius}(C) / \sqrt{2}$, which is at least $k_{1}(c) f(x) / \sqrt{2}$ by (i). The angle of any wedge of $E$ and $F$ is at least $\alpha_{m}$. Hence, the distance between $x$ and $E$ is at least $\frac{\sin \alpha_{m}}{\sqrt{2}} k_{1}(c) f(x)=k_{2}(c) f(x)$.

\subsection{Termination and mesh quality}

In this section, we prove that the inter-vertex distances are above certain thresholds as QMESH inserts vertices. This allows to prove the termination of QMESH by a packing argument. We then show that any remaining skinny tetrahedron is close to some sharp vertex or edge.

The lower bounds on inter-vertex distances in the InITIALIZE and CONFORM phases will be directly related to local feature sizes, while the REFINE phase will be handled using an inductive argument. The inductive argument makes use of a parent-child relation defined as follows.

Let $x$ be a vertex inserted or rejected by QMesH. The parent of $x$ is an input vertex or a vertex inserted or rejected by QMesh. If $x$ is a vertex of $\mathcal{P}$ or a vertex inserted during InITIALIZE, its parent is undefined. Otherwise, the parent $p$ is defined as follows.

- Suppose that $x$ splits a subsegment (shield or non-shield) or a subfacet $h$. Let $B$ be the diametric ball of $h$. If $B$ contains some vertex in $\mathcal{V}, p$ is the encroaching vertex nearest to $x$. If $B$ is empty and QMESH is going to reject a vertex inside $B$ for encroaching $h, p$ is that vertex.

- If $x$ is the circumcenter of a skinny tetrahedron $\tau, p$ is one of the endpoints of the shortest edge of $\tau$. Between the two endpoints of the shortest edge, $p$ is the one that appears in $\mathcal{V}$ later.

It is still possible that $x$ has no parent. This happens when $x$ is inserted to split a subsegment in rule 3 . When a subfacet is split by $x$ in rule $3, x$ may or may not have a parent. If the diametric ball of the subfacet is empty, $x$ does not have any parent. But, if this ball is not empty, $x$ has a parent, namely the vertex in $\mathcal{V}$ nearest to $x$. In the case where $x$ is the circumcenter of a subfacet $h$, if the insertion of $x$ is triggered by the circumcenter of a skinny tetrahedron in rule 6 , the parent of $x$ is not necessarily the triggering point. This is because the diametric ball of $h$ may already contain some vertices in $\mathcal{V}$.

The insertion radius $r_{x}$ of $x$ is the distance from $x$ to its nearest neighbor in the current $\mathcal{V}$ when $x$ is inserted or rejected. So it follows from definition that if $x$ is an input vertex, then $r_{x} \geq f(x)$.

In Sections 4.3.1-4.3.3, we bound the insertion radii from below in each phase, which leads to the proof in Section 4.3.4 that QMESH terminates. We end this preamble with a technical result concerning insertion radius.

Lemma 4.11 Let $x$ be a vertex inserted or rejected by QMESH. Assume that its parent $p$ is defined. If $p$ is a vertex in $\mathcal{V}$, then $r_{x}=\|p-x\|$. Otherwise, the following hold: 
(i) If $x$ splits a non-shield subsegment, subfacet, or tetrahedron, then $r_{x}$ is equal to the radius of the diametric ball of the subsegment or subfacet, and $r_{x} \geq\|p-x\|$.

(ii) If $x$ splits a shield subsegment, then $r_{x}$ is at least $(1-\tan (\pi / 8))$ times the radius of the diametric ball of the subsegment, and $r_{x} \geq \frac{1-\tan (\pi / 8)}{1+\tan (\pi / 8)}\|p-x\|$.

Proof. Let $\sigma$ be the subsegment, subfacet, or tetrahedron split by $x$. Let $B$ be the diametric ball of $\sigma$. If $p$ is a vertex in $\mathcal{V}$, it follows from definition that $r_{x}=\|p-x\|$. Otherwise, we know that $B$ does not strictly contain any vertex.

If $\sigma$ is a non-shield subsegment or subfacet, then $x$ is the center of $B$ and QMESH rejects $p$ for lying inside $B$. So $r_{x}=\operatorname{radius}(B) \geq\|p-x\|$. If $\sigma$ is a tetrahedron, then $x$ is the center of $B$ and $p$ lies on the boundary of $B$. So $r_{x}=\operatorname{radius}(B)=\|p-x\|$. Suppose that $\sigma$ is a shield subsegment. The difference from the previous cases is that $x$ is not the center of $B$. Let $y$ be the center of $B$. Let $\widehat{v}$ be the vertex ball that contains $\sigma$. The emptiness of $B$ implies that $r_{x}$ is at least the distance from $x$ to the boundary of $B$, which is

$$
\operatorname{radius}(B)-\|x-y\| \text {. }
$$

Since the parent $p$ is defined, $x$ is not inserted in the Initialize phase. So $\sigma$ corresponds to a sector at $v$ with angle less than $\pi$. Moreover, $v$ cannot lie inside $B$ as $B$ is empty. Thus $\sigma$ subtends at $v$ an angle $\theta \leq \pi / 2$. This implies that

$$
\|x-y\|=\operatorname{radius}(B) \tan (\theta / 4) \leq \operatorname{radius}(B) \tan (\pi / 8) .
$$

Substituting (4.4) into (4.3) yields $r_{x} \geq(1-\tan (\pi / 8)) \operatorname{radius}(B)$. Also, (4.4) implies that $\|p-x\| \leq \operatorname{radius}(B)+\|x-y\| \leq(1+\tan (\pi / 8)) \operatorname{radius}(B)$. It follows that $r_{x} \geq(1-$ $\tan (\pi / 8)) \operatorname{radius}(B) \geq \frac{1-\tan (\pi / 8)}{1+\tan (\pi / 8)}\|p-x\|$.

\subsubsection{Initialization}

Lemma 4.12 During Initialize, for each vertex $x \in \mathcal{V}, r_{x} \geq \frac{\sin \alpha_{m}}{5} f(x)$.

Proof. If $x$ is an input vertex, it follows from definition that $r_{x} \geq f(x)$. Suppose that $x$ is a vertex inserted by QMesh. During Initialize, all vertices inserted lie on the boundaries of vertex balls. So $x$ lies on $\widehat{v}$ for some sharp vertex $v$. The Lipschitz condition implies that $f(x) \leq f(v)+\|v-x\| \leq 5 f(v) / 4$. By Lemma 3.1(ii) and (iii), the nearest neighbor of $x$ is contained in $\widehat{v}$.

Suppose that $x$ is the intersection between an incident edge of $v$ and the boundary of $\widehat{v}$. Then the vertices inserted so far are intersections between edges and boundaries of vertex balls. So either the nearest neighbor of $x$ is $v$ or it lies on another incident edge of $v$. In the first case, $r_{x}=f(v) / 4 \geq f(x) / 5$. In the second case, $r_{x} \geq f(v) \sin \alpha_{m} / 4 \geq f(x) \sin \alpha_{m} / 5$.

The remaining possibility is that $x$ is inserted to split a shield subsegment $a b$ because $a b$ corresponds to a sector with angle $\pi$ or more. It is clear that $v$ is the nearest neighbor of $x$ in this case. So $r_{x}=f(v) / 4 \geq f(x) / 5$. 


\subsubsection{Conformation}

We first prove a lower bound on the insertion radius of a vertex that splits a subsegment.

Lemma 4.13 Let $x$ be the vertex inserted to split a subsegment during Conform. Then $r_{x}=$ $\Omega(f(x))$.

Proof. Let $a b$ be the subsegment split by $x$. Let $p$ be the point that encroaches $a b$ and triggers the insertion of $x$. Notice that during Conform, all vertices in $\mathcal{V}$ lie on the elements of $\mathcal{P}$.

If $p$ is a subsegment endpoint or the midpoint of a sharp subsegment, Lemma 4.3 implies that $r_{x}=\Omega(f(x))$. The remaining possibility is that $p$ is the circumcenter of a subfacet $h$. QMesh attempts to insert $p$, but rejects $p$ eventually for encroaching $a b$. It follows that $p$ is the parent of $x$ and no subsegment is encroached before the attempted insertion of $p$. Let $F$ be the facet containing $h$. Let $r$ be the circumradius of $h$.

Case 1: $h \in \operatorname{Del} \mathcal{V}$. In this case, QMesh tries to insert $p$ in rule 3. Rule 3 applies because the diametric ball of $h$ intersects a sharp edge disjoint from $F$. So $r \geq f(p)$.

Case 1.1: Neither $a$ nor $b$ lies inside the diametric ball of $h$. By Lemma 4.11, whether $a b$ is shield or not, $r_{x}$ is asymptotically bounded from below by the radius of the diametric ball of $a b$. So $r_{x}=\Omega(\|a-b\|)$. Since $p$ lies on or inside the diametric ball of $a b$, we have $\|a-b\| / 2 \geq r / \sqrt{2}$. So $r_{x}=\Omega(r)=\Omega(f(p))$. Also, by Lemma 4.11, $r_{x}=\Omega(\|p-x\|)$ whether $a b$ is shield or not. Thus the Lipschitz condition implies that $f(x) \leq f(p)+\|p-x\|=O\left(r_{x}\right)$.

Case 1.2: $a$ or $b$ lies inside the diametric ball of $h$. The emptiness of the circumcircle of $h$ implies that $a b$ does not lie on $F$ (including the boundary of $F$ ). Since the diametric ball of $h$ strictly contains $a$ or $b$, which lies on some element of $\mathcal{P}$, by shifting the ball center slightly, we obtain a circumball $B$ of $h$ where $\operatorname{radius}(B) \geq r \geq f(p)$, and $B$ satisfies the conditions of Lemma 4.10. By the definition of SpliTF, $p$ does not encroach any subsegment in $\partial F$ in order to cause the splitting of $a b$. Thus Lemma 4.10(ii) implies that $\|p-x\| \geq k_{2}(1) f(p)$. The Lipschitz condition implies that $f(x) \leq f(p)+\|p-x\| \leq \frac{1+k_{2}(1)}{k_{2}(1)}\|p-x\|$. By Lemma 4.11, $\|p-x\|=O\left(r_{x}\right)$ whether $a b$ is shield or not. It follows that $f(x)=O\left(r_{x}\right)$.

Case 2: $h \notin \operatorname{Del} \mathcal{V}$. By Lemma 4.9, the conditions of Lemma 4.10 are satisfied.

Case 2.1: $a b$ lies on $F$ (including the boundary of $F$ ). The emptiness of the circumcircle of $h$ implies that neither $a$ nor $b$ lies inside the diametric ball of $h$. By Lemmas 4.9 and 4.10(i), $r \geq k_{1}\left(\lambda_{2}\right) f(p)$. Then we can use the analysis in case 1.1 to obtain $r_{x}=\Omega(f(x))$.

Case 2.2: $a b$ does not lie on $F$ (including the boundary of $F$ ). By the definition of SplitF, $p$ does not encroach any subsegment in $\partial F$ in order to cause the splitting of $a b$. So $\|p-x\| \geq k_{2}\left(\lambda_{2}\right) f(p)$ by Lemmas 4.9 and 4.10(ii). The Lipschitz condition implies that $f(x) \leq f(p)+\|p-x\| \leq \frac{1+k_{2}\left(\lambda_{2}\right)}{k_{2}\left(\lambda_{2}\right)}\|p-x\|$. By Lemma 4.11, $\|p-x\|=O\left(r_{x}\right)$ whether $a b$ is shield or not. It follows that $f(x)=O\left(r_{x}\right)$. 
We are ready to give the full analysis of the lower bounds on inter-vertex distances at the end of the CONFORM phase. We also show that each point on a sharp subsegment lies inside a protecting ball that is not too small.

Lemma 4.14 At the end of CONFORM, there are constants $\lambda_{3}, \lambda_{4}>0$ such that

(i) For any vertex $x \in \mathcal{V}, r_{x} \geq \lambda_{3} f(x)$.

(ii) For any point $z$ on a sharp subsegment, a ball centered at $z$ with radius $\lambda_{4} f(z)$ lies inside some protecting ball.

Proof. We first prove (i). By Lemma 4.12, for any vertex $x$ inherited from the InITIALIzE phase, $r_{x} \geq\left(\sin \alpha_{m} / 5\right) f(x)$. Consider the insertion of a vertex $x$ during Conform. If $x$ splits a subsegment, then $r_{x}=\Omega(f(x))$ by Lemma 4.13. Suppose that $x$ is the circumcenter of a subfacet $h$ on a facet $F$. Thus no subsegment is encroached before the insertion of $x$, and so $x$ lies in the interior of $F$. The vertex $x$ does not encroach any subsegment; otherwise, $x$ would be rejected.

If $h \notin \operatorname{Del} \mathcal{V}$, then $r_{x} \geq k_{2}\left(\lambda_{2}\right) f(x)$ by Lemmas 4.9 and 4.10. Suppose that $h \in \operatorname{Del} \mathcal{V}$. So $x$ is inserted by rule 3 . Rule 3 applies because the diametric ball of $h$ intersects a sharp edge disjoint from $F$. It follows that $r \geq f(x)$, where $r$ is the circumradius of $h$. There are several cases to consider.

Case 1: $x$ has no parent. Then the diametric ball of $h$ is empty and so $r_{x} \geq r \geq f(x)$.

Case 2: $x$ has a parent $p$. So $p$ is a vertex in $\mathcal{V}$ inside the diametric ball of $h$. Thus $r_{x}=\|p-x\|$. Recall that all vertices in $\mathcal{V}$ in the CONFORm phase lie on the elements of $\mathcal{P}$. So $p$ lies on some facet $E$ (including the boundary of $E$ ). By Lemma 4.8, $E$ is not coplanar with $F$.

If $E$ is disjoint from $F$, then $r_{x}=\|p-x\| \geq f(x)$. Suppose that $E$ and $F$ are adjacent. Let $q$ be the nearest point to $x$ on the intersection line of the support planes of $E$ and $F$.

Case 2.1: $q \notin E \cap F$. If the wedge of $E$ and $F$ containing $p$ and $x$ is non-sharp, then $r_{x}=\|p-x\|=\Omega(f(x))$ by Lemma 4.7(ii). Otherwise, $r_{x}=\|p-x\|=\Omega(f(x))$ by Lemma 4.7(iii).

Case 2.2: $q \in E \cap F$. We claim that $\|q-x\| \geq r / \sqrt{2}$. Let $C$ denote the circumcircle of $h$. If $q$ does not lie inside $C$, then $\|q-x\| \geq r$. Otherwise, the emptiness of $C$ implies that $q$ lies on a subsegment $s$ in $E \cap F$, and $s$ crosses $C$ completely. In order that $x$ does not encroach $s$, the distance from $x$ to $s$ must be at least $r / \sqrt{2}$. This proves our claim. Since the angle of any wedge of $E$ and $F$ is at least $\alpha_{m}$, the claim implies that $r_{x}=\|p-x\| \geq\|q-x\| \sin \alpha_{m} \geq \frac{\sin \alpha_{m}}{\sqrt{2}} \cdot r \geq \frac{\sin \alpha_{m}}{\sqrt{2}} f(x)$.

This finishes the proof of (i).

Consider (ii). Let $m$ be the midpoint of a sharp subsegment $a b$. For any point $z \in a m$, the ball centered at $z$ with radius $\|a-m\|$ lies inside the protecting ball of $a b$. The Lipschitz condition implies that

$$
f(z) \leq f(a)+\|a-z\| \leq f(a)+\|a-m\|
$$


Next, we relate $f(a)$ and $\|a-m\|$ to $f(z)$. We claim that $\|a-b\| \geq \frac{\lambda_{3}}{1+\lambda_{3}} f(a)$. If $a$ was not inserted before $b$, then $\|a-b\| \geq r_{a}$ which is at least $\lambda_{3} f(a)$ by (i). If $a$ was inserted before $b$, we have $\|a-b\| \geq \lambda_{3} f(b)$ similarly. Then the Lipschitz condition implies that $f(a) \leq f(b)+\|a-b\| \leq \frac{1+\lambda_{3}}{\lambda_{3}}\|a-b\|$. Our claim implies that $\|a-m\| \geq \frac{\lambda_{3}}{2+2 \lambda_{3}} f(a)$. Substituting this into (4.5), we get $f(z) \leq \frac{2+3 \lambda_{3}}{\lambda_{3}}\|a-m\|$. Since the ball centered at $z$ with radius $\|a-m\|$ lies inside the protecting ball of $a b$, decreasing its radius to $\frac{\lambda_{3}}{2+3 \lambda_{3}} f(z)$ keeps it inside.

\subsubsection{Refinement}

The main difference of the REFINE phase from the CONFORM phase is that skinny tetrahedra are split in the REFinE phase. As in the case of standard Delaunay refinement analysis, we are to analyze the effect of splitting skinny tetrahedra on insertion radii using an inductive argument. However, there are issues that have to be addressed first. Due to the splitting of skinny tetrahedra, some vertices may not lie on the elements of $\mathcal{P}$. This means that Lemma 4.9(iii) does not apply. So we cannot borrow the proof of Lemma 4.14(i) for the Conform phase to conclude that the insertion radii of circumcenters of subfacets are also large in the REFINE phase. Furthermore, unlike in the CONFORM phase, we need to look at circumcenters rejected by QMesh as well for the subsequent inductive argument to work. We first tackle these issues in the next two lemmas. Notice that when QMESH inserts or rejects a vertex in the REFINE phase, its parent is always defined.

Lemma 4.15 Let $h$ be a subfacet on a facet $F$. Let $C$ and $x$ be the circumcircle and circumcenter of $h$, respectively. Suppose that $x$ projects orthogonally to a point $y$ on a sharp edge in $\partial F$ such that $\|x-y\| \leq \lambda_{4} f(x) /\left(3+\lambda_{4}\right)$, where $\lambda_{4}$ is the constant in Lemma 4.14(ii). Then the following hold:

(i) $f(y) \geq 3 f(x) /\left(3+\lambda_{4}\right)$.

(ii) $\lambda_{4} f(y) \geq 3\|x-y\|$.

(iii) If $h \notin \operatorname{Del} \mathcal{V}$, then $\operatorname{radius}(C)=\Omega(f(x))$.

Proof. Substituting the inequality $\|x-y\| \leq \lambda_{4} f(x) /\left(3+\lambda_{4}\right)$ into $f(y) \geq f(x)-\|x-y\|$ proves (i) and (ii). Consider (iii). First, observe that $h$ is encroached by a vertex of $\mathcal{V}$ since otherwise $h$ would have appeared in Del $\mathcal{V}$. All encroaching vertices of $h$ must lie on elements of $\mathcal{P}$ as vertices not lying on $\mathcal{P}$ (circumcenters of tetrahedra) and encroaching a subfacet are rejected in rule 6. Now we have all conditions of Lemma 4.9 in place. So, there is a circumball $B$ of $h$ such that $B$ contains two vertices and one of them, say $v$, lies on the same side of $h$ as the center of $B$. If $v$ also lies on some element of $\mathcal{P}$, by Lemmas $4.9(\mathrm{iii})$ and $4.10(\mathrm{i}), \operatorname{radius}(C) \geq k_{1}\left(\lambda_{2}\right) f(x)$ and we are done. Suppose that $v$ does not lie on any element of $\mathcal{P}$. So $v$ was inserted as the circumcenter of a skinny tetrahedron. By Lemma 4.14(ii), a protecting ball contains the ball centered at $y$ with radius $\lambda_{4} f(y)$. This implies that $v$ could not be inserted inside it, i.e., 
$\|v-y\| \geq \lambda_{4} f(y)$. Thus

$$
\begin{aligned}
\|v-x\| & \geq\|v-y\|-\|x-y\| \\
& \stackrel{(\text { ii) }}{\geq} 2 \lambda_{4} f(y) / 3 \\
& \stackrel{\text { (i) }}{\geq} 2 \lambda_{4} f(x) /\left(3+\lambda_{4}\right) .
\end{aligned}
$$

By the triangle inequality, $\operatorname{radius}(B)$ is at least $\|v-x\|$ minus the distance between $x$ and the center of $B$. So Lemma $4.10(i)$ applies and yields $\operatorname{radius}(C) \geq k_{1}\left(\frac{2 \lambda_{4}}{3+\lambda_{4}}\right) f(x)$.

Lemma 4.16 Assume that no subsegment is encroached by a vertex. Let $x$ be the circumcenter of a subfacet inserted or rejected during REFINE. Let $p$ be the parent of $x$. If $p$ is a vertex in $\mathcal{V}$ lying on some element of $\mathcal{P}$, then $r_{x} \geq \lambda_{5} f(x)$ for some constant $\lambda_{5}=\Theta\left(\sin \alpha_{m}\right)$.

Proof. Let $h$ be the subfacet of which $x$ is the circumcenter. Let $F$ be the facet of $\mathcal{P}$ that contains $h$. Let $F^{\prime}$ be a facet containing $p$ (including the boundary of $F^{\prime}$ ). By Lemma $4.8, F$ and $F^{\prime}$ are non-coplanar.

If $F$ and $F^{\prime}$ are disjoint, then $r_{x}=\|p-x\| \geq f(x)$. Suppose that $F$ and $F^{\prime}$ are adjacent. If $p$ and $x$ lie on a non-sharp wedge of $F$ and $F^{\prime}$, then $r_{x}=\|p-x\|=\Omega(f(x))$ by Lemma 4.7(ii). From now on, we assume that $p$ and $x$ lie on a sharp wedge of $F$ and $F^{\prime}$. Let $y$ be the closest point to $x$ on the intersection line of the support planes of $F$ and $F^{\prime}$.

Case 1: $y$ lies on an edge in $F \cap F^{\prime}$ (including the edge endpoints). If the edge is non-sharp, then $\|p-x\|=\Omega(f(x))$ by Lemma 4.7(iii). Suppose that the edge is sharp. Let $W$ be the wedge of $F$ and $F^{\prime}$ containing $p$ and $x$. Recall that $W$ is sharp by assumption. Since the angle of $W$ is at least $\alpha_{m}$, we have

$$
\|p-x\| \geq\|x-y\| \sin \alpha_{m}
$$

Also, since $\angle p y x$ is at least the angle of $W$ by Lemma 2.1 , we have

$$
\|p-x\| \geq\|p-y\| \sin \angle p y x \geq\|p-y\| \sin \alpha_{m} .
$$

There are two cases depending on the length of $\|x-y\|$. Let $C$ be the circumcircle of $h$. Let $\lambda_{4}$ be the constant in Lemma 4.14(ii).

Case 1.1: $\|x-y\| \geq \operatorname{radius}(C) / 2$. If $\|x-y\| \geq \lambda_{4} f(x) /\left(3+\lambda_{4}\right)$, then by (4.6), $r_{x}=$ $\|p-x\|=\Omega(f(x))$ and we are done.

Suppose that $\|x-y\|<\lambda_{4} f(x) /\left(3+\lambda_{4}\right)$. Since radius $(C) \leq 2\|x-y\|$ by assumption, Lemma 4.15(ii) implies that $\lambda_{4} f(y) \geq\|x-y\|+\operatorname{radius}(C)$. Combining this with Lemma 4.14(ii), we conclude that the diametric ball of $h$ lies inside some protecting ball. QMESH never inserts the circumcenter of a tetrahedron inside any protecting ball. It follows that QMESH attempts to insert $x$ in rule 5 because $h \notin \operatorname{Del} \mathcal{V}$. Hence, Lemma 4.15(iii) implies that $\operatorname{radius}(C)=\Omega(f(x))$. So $\|x-y\| \geq \operatorname{radius}(C) / 2=$ $\Omega(f(x))$. Substituting this into (4.6) yields $r_{x}=\|p-x\|=\Omega(f(x))$. 
Case 1.2: $\|x-y\|<\operatorname{radius}(C) / 2$. Recall that it is the assumption of case 1 that $y$ lies on an edge in $F \cap F^{\prime}$. By the emptiness of $C$, a subsegment on this edge crosses $C$ completely. Clip this subsegment within $C$ to obtain a chord $s$. Notice that $y$ is the midpoint of $s$. Since $\|x-y\|<\operatorname{radius}(C) / 2$, we have the following two inequalities:

$$
\begin{gathered}
\operatorname{length}(s) \geq \sqrt{3} \operatorname{radius}(C), \\
\operatorname{length}(s) \geq\|x-y\|+\operatorname{radius}(C) .
\end{gathered}
$$

Since $p$ does not encroach the subsegment containing $s, p$ lies outside the diametric ball of $s$. It follows that

$$
\|p-y\| \geq \operatorname{length}(s) / 2 \stackrel{(4.8)}{\geq} \sqrt{3} \operatorname{radius}(C) / 2 .
$$

If radius $(C) \geq 2 \lambda_{4} f(x) /\left(3+\lambda_{4}\right)$, then (4.10) and (4.7) imply that $r_{x}=\|p-x\|=$ $\Omega(f(x))$ and we are done.

Suppose that radius $(C)<2 \lambda_{4} f(x) /\left(3+\lambda_{4}\right)$. It follows that $\|x-y\|<\lambda_{4} f(x) /\left(3+\lambda_{4}\right)$. The inequality (4.9) implies that if we double the size of the diametric ball of $s$, it contains the diametric ball of $h$. Therefore, the diametric ball of $h$ lies inside some protecting ball. As in case 1.1, we conclude that $h \notin \operatorname{Del} \mathcal{V}$ and so Lemma 4.15(iii) implies that radius $(C)=\Omega(f(x))$. Then (4.10) and (4.7) imply that $r_{x}=\|p-x\|=$ $\Omega(f(x))$.

Case 2: $y$ is an isolated vertex in $F \cap F^{\prime}$. Then $r_{x}=\|p-x\|=\Omega(f(x))$ by Lemma 4.5(iii).

Case 3: $y \notin F \cap F^{\prime}$. If the wedge of $F$ and $F^{\prime}$ containing $p$ and $x$ is non-sharp, we apply Lemma 4.7(ii); otherwise, we apply Lemma 4.7(iii). In both cases, we conclude that $r_{x}=\|p-x\|=\Omega(f(x))$.

We are ready to apply induction to show that in the REFINE phase, the insertion radius of any vertex inserted or rejected is bounded from below by its local feature size.

Lemma 4.17 If $x$ exists in $\mathcal{V}$ before the invocation of REFINE or $x$ is inserted or rejected by QMesh during REFINE, then $r_{x}=\Omega(f(x))$.

Proof. If $x$ exists in $\mathcal{V}$ before the invocation of REFInE, then $r_{x} \geq \lambda_{3} f(x)$ by Lemma 4.14(i). Assume that $x$ is inserted or rejected during Refine. We define the type of $x$ as follows. If $x$ splits a shield or non-shield subsegment, $x$ has type 4 . If $x$ is the circumcenter of a subfacet, $x$ has type 5 . If $x$ is the circumcenter of a tetrahedron, $x$ has type 6 . We prove a stronger statement by induction: if $x$ has type $i$, then $r_{x} \geq f(x) / C_{i}$ for some constants $C_{4}>C_{5}>C_{6}>1$. In the induction steps, we develop several inequalities involving $C_{4}, C_{5}$, and $C_{6}$ that need to hold for the proof. We set the values of $C_{4}, C_{5}$, and $C_{6}$ at the end to satisfy all the inequalities.

Let $p$ be the parent of $x$. Suppose that $r_{x} \geq c \cdot r_{p}$ for some constant $c$. By Lemma 4.11, if $x$ has type 5 or 6 , then $r_{x} \geq\|p-x\|$ and so

$$
\frac{f(x)}{r_{x}} \leq \frac{f(p)}{r_{x}}+\frac{\|p-x\|}{r_{x}} \leq \frac{f(p)}{r_{x}}+1 \leq \frac{f(p)}{c \cdot r_{p}}+1 .
$$


Otherwise, $x$ has type 4 and $r_{x} \geq \frac{1-\tan (\pi / 8)}{1+\tan (\pi / 8)}\|p-x\|$. So

$$
\frac{f(x)}{r_{x}} \leq \frac{f(p)}{r_{x}}+\frac{\|p-x\|}{r_{x}} \leq \frac{f(p)}{r_{x}}+\frac{1+\tan (\pi / 8)}{1-\tan (\pi / 8)} \leq \frac{f(p)}{c \cdot r_{p}}+\frac{1+\tan (\pi / 8)}{1-\tan (\pi / 8)} .
$$

Case 1: $x$ has type 4. If $p$ has type $4, p$ must be inserted and it is an endpoint of a subsegment (shield or non-shield). By Lemma 4.3, $r_{x} \geq \lambda_{6} f(x)$ for some constant $\lambda_{6}>0$.

Suppose that $p$ has type 5 or 6 . Since $p$ encroaches the subsegment split by $x$, the radius of the diametric ball of this subsegment is at least $r_{p} / \sqrt{2}$. By Lemma $4.11, r_{x} \geq \frac{1-\tan (\pi / 8)}{\sqrt{2}} \cdot r_{p}$. As $C_{5}>C_{6}$, by induction assumption, $r_{p} \geq f(p) / C_{5}$ regardless of the type of $p$. This gives $\frac{f(x)}{r_{x}} \leq \frac{\sqrt{2}}{1-\tan (\pi / 8)} \cdot \frac{f(p)}{r_{p}}+\frac{1+\tan (\pi / 8)}{1-\tan (\pi / 8)} \leq \frac{\sqrt{2}}{1-\tan (\pi / 8)} \cdot C_{5}+\frac{1+\tan (\pi / 8)}{1-\tan (\pi / 8)}$.

So the inequalities $\beta_{1} C_{5}+\beta_{2} \leq C_{4}$ and $C_{4} \geq 1 / \lambda_{6}$ need to hold, where $\beta_{1}=\frac{\sqrt{2}}{1-\tan (\pi / 8)}$ and $\beta_{2}=\frac{1+\tan (\pi / 8)}{1-\tan (\pi / 8)}$.

Case 2: $x$ has type 5. If $p$ is a vertex lying on some element of $\mathcal{P}$, Lemma 4.16 implies that $r_{x} \geq \lambda_{5} f(x)$. Otherwise, $p$ must have type 6 . Let $h$ be the subfacet split by $x$. Let $F$ be the facet containing $h$.

We claim that $p$ is not a vertex in $\mathcal{V}$. Suppose not. Then $p$ was inserted at certain time $t$ in the past. We first prove that the diametric ball of $h$ is contained in the union of the diametric balls of subsegments and subfacets on $F$ (including its boundary) at time $t$.

Go back to time $t$. It suffices to show that for any point $q$ inside the diametric ball of $h, q$ lies inside the diametric ball of some subsegment or subfacet on $F$ at time $t$. Call the disk circumscribing a triangle its circumdisk and the smallest disk circumscribing a segment its diametric disk. For triangles, Observe that the circumdisk of $h$ is also empty at time $t$ and since $p$ is to be inserted, no subsegment in $\partial F$ is encroached.

Since $x$ lies inside $F$, we argue that the circumdisk of $h$ is contained in the union of the diametric disks of subsegments and circumdisks of subfacets on $F$ at time $t$. Let $q^{\prime}$ be the orthogonal projection of $q$ onto the plane of $F$. Suppose that $q^{\prime}$ lies inside some subfacet $\sigma$ on $F$ at time $t$. If the circumdisk of $h$ is contained inside the circumdisk of $\sigma$, the diametric ball of $\sigma$ clearly contains the diametric ball of $h$. Otherwise, the boundaries of the diametric balls of $\sigma$ and $h$ intersect at a circle and let $H$ be the plane that contains this circle. Since the circumdisk of $h$ does not contain the vertices of $\sigma, \sigma$ and hence $q^{\prime}$ lie on one side of $H$. Moreover, the diametric ball of $\sigma$ contains the part of the diametric ball of $h$ on this side of $H$. It follows that $q$ lies inside the diametric ball of $\sigma$. The remaining possibility is that $q^{\prime}$ lies outside $F$. In this case, $q^{\prime}$ must lie inside the diametric disk of some subsegment in $\partial F$ at time $t$. Then a similar argument shows that $q$ lies inside the diametric ball of this subsegment.

Since the diametric ball of $h$ is contained in the union of the diametric balls of subsegments and subfacets on $F$ at time $t, p$ must encroach upon some subsegment or subfacet when it was inserted. But then QMESH should have rejected $p$, a contradiction. This completes the proof that $p$ is not a vertex in $\mathcal{V}$.

Hence, we can infer that QMesh tries to insert $p$ to split a skinny tetrahedron, but then it rejects $p$ for encroaching $h$. And the diametric ball $B$ of $h$ is empty. Since $p$ lies inside $B, \operatorname{radius}(B) \geq r_{p} / 2$. Thus $r_{x}=\operatorname{radius}(B) \geq r_{p} / 2$. Since $r_{p} \geq f(p) / C_{6}$ by induction assumption, we have $\frac{f(x)}{r_{x}} \leq \frac{2 f(p)}{r_{p}}+1 \leq 2 C_{6}+1$. So the inequalities $2 C_{6}+1 \leq C_{5}$ and $C_{5} \geq 1 / \lambda_{5}$ need to hold. 
Case 3: $x$ has type 6. Let $\tau$ be the skinny tetrahedron of which $x$ is the circumcenter. Observe that $r_{p}$ is at most the shortest edge length of $\tau$. This is because the other endpoint of this edge was already in $\mathcal{V}$ when $p$ was inserted; refer to the definition of $p$. This implies that $r_{x}=\|p-x\| \geq \rho_{0} r_{p}$.

If $p$ exists as a vertex in $\mathcal{V}$ before the invocation of Refine, then $r_{p} \geq \lambda_{3} f(p)$ by Lemma 4.14(i). So $r_{x} \geq \rho_{0} \lambda_{3} f(p)$. The Lipschitz condition implies that $f(x) \leq f(p)+$ $\|p-x\| \leq \frac{\rho_{0} \lambda_{3}+1}{\rho_{0} \lambda_{3}} \cdot r_{x}$.

Suppose that $p$ is inserted into $\mathcal{V}$ during Refine. As $C_{4}>C_{5}>C_{6}$, by induction assumption, $r_{p} \geq f(p) / C_{4}$ regardless of the type of $p$. This gives $\frac{f(x)}{r_{x}} \leq \frac{f(p)}{\rho_{0} \cdot r_{p}}+1 \leq \frac{C_{4}}{\rho_{0}}+1$.

So the inequalities $C_{4} / \rho_{0}+1 \leq C_{6}$ and $C_{6} \geq \frac{\rho_{0} \lambda_{3}+1}{\rho_{0} \lambda_{3}}$ need to hold.

We set $C_{6}=\max \left\{\frac{\rho_{0}+\beta_{1}+\beta_{2}}{\rho_{0}-2 \beta_{1}}, \frac{1}{\lambda_{5}}, \frac{1}{\lambda_{6}}, \frac{\rho_{0} \lambda_{3}+1}{\rho_{0} \lambda_{3}}\right\}, C_{5}=2 C_{6}+1$, and $C_{4}=\beta_{1} C_{5}+\beta_{2}$. Notice that $C_{i}>0$ for $i=4,5,6$ since $\rho_{0}>2 \beta_{1}=\frac{2 \sqrt{2}}{1-\tan (\pi / 8)}$ as chosen by QMEsH.

The above proof shows that $C_{6}$ is dependent on $\rho_{0}$ and $\alpha_{m}$ because $\lambda_{i}, i=3,5,6$, depend on $\alpha_{m}$. For example, if we choose $\rho_{0}$ to be 6 , we get $C_{6}=\max \left\{9.25, \Theta\left(\frac{1}{\alpha_{m}}\right), \Theta\left(\frac{1}{\sin ^{2} \alpha_{m} \tan \alpha_{m}}\right)\right\}$. Therefore, both $C_{5}$ and $C_{4}$ also have similar dependencies on $\rho_{0}$ and $\alpha_{m}$. We observe that if one can lower $\rho_{0}$, it perhaps improves the constants $C_{i}$ s given that $\alpha_{m}$ is sufficiently large. This indicates a tradeoff between the quality of tetrahedra and the size of the output.

Our bound for $\rho_{0}$ is larger than the bound of 2 for standard Delaunay refinement. This is due to two reasons. First, in case 1 of the proof of Lemma 4.17, we have $r_{x} \geq \frac{1-\tan (\pi / 8)}{\sqrt{2}} r_{p}$. Second, in case 2 of the proof of Lemma 4.17, we have $r_{x} \geq r_{p} / 2$. The product of the constant factors in these two inequalities yields the threshold $2 \sqrt{2} /(1-\tan (\pi / 8))$ for $\rho_{0}$. This threshold can be lowered as follows. First, the factor $1-\tan (\pi / 8)$ comes from Lemma 4.11(ii). If we split all shield subsegments in Initialize until they subtend an angle at most $\theta$ for some constant $\theta<\pi / 2$, the factor becomes $1-\tan (\theta / 4)$ instead. This will worsen the output angles at the sharp vertices though. Second, the inequality $r_{x} \geq r_{p} / 2$ in case 2 of the proof of Lemma 4.17 can be improved to $r_{x} \geq r_{p} / \sqrt{2}$ if we do not split just any encroached subfacet, but the one that contains the projection of the encroaching vertex [17]. Hence, the threshold for $\rho_{0}$ can be lowered to $2 /(1-\tan (\theta / 4))$ for any $\theta<\pi / 2$. In other words, it can be reduced to $2+\epsilon$ for any fixed $\epsilon>0$.

\subsubsection{Finale}

Theorem 4.1 QMesh terminates and returns a Delaunay mesh such that for any two mesh vertices $a$ and $b,\|a-b\|=c(\max \{f(a), f(b)\})$ where $c=\Theta\left(\sin ^{2} \alpha_{m}\right)$. The vertices of any skinny tetrahedron are at distance $O(f(x))$ from some sharp vertex $x$ or some point $x$ on a sharp edge.

Proof. For any two vertices $a, b \in \mathcal{V}$ such that $a$ appears in $\mathcal{V}$ not earlier than $b,\|a-b\| \geq$ $\mu f(a)$ for some constant $\mu>0$ (Lemma 4.17). One can work backwards through different lemmas to assert $\mu=\Theta\left(\sin ^{2} \alpha_{m}\right)$. So $f(b) \leq f(a)+\|a-b\| \leq \frac{1+\mu}{\mu}\|a-b\|$. In all, $\|a-b\|=$ $\Omega(\max \{f(a), f(b)\})$. A packing argument within the domain bounded by $\mathcal{B}$ shows that QMESH terminates. 
Let $\tau$ be a skinny tetrahedron. Let $R$ be the circumradius of $\tau$. Note that the circumcenter $q$ lies inside some protecting ball $B$. If $B$ is the vertex ball at a sharp $v$, then $f(v) / 4 \geq\|q-v\| \geq R$. So the vertices of $\tau$ are at distance $f(v) / 4+R \leq f(v) / 2$ from $v$. If $B$ is not a vertex ball, $B$ is twice as large as the diametric ball $B_{a b}$ of some sharp subsegment $a b$ at the end of Conform. Let $x$ be the midpoint of $a b$. Let $X$ denote $\|a-b\| / 2$. It suffices to show that $X \leq \sqrt{2} f(x)$.

Assume to the contrary that $X>\sqrt{2} f(x)$. Then $B_{a b}$ intersects an edge or a facet of $\mathcal{P}$ disjoint from the edge containing $a b$. Let $E$ be such an element closest to $x$. Since $a b$ is not encroached at the end of CONFORM, no vertex of $E$ lies inside $B_{a b}$. It follows that $x$ projects orthogonally onto a point $y$ in the interior of $E$. Let $h$ be the subsegment or subfacet on $E$ that contains $y$. Let $w$ be the vertex of $h$ closest to $y$. Since $\|w-x\| \geq X>\sqrt{2} f(x)$ and $\|x-y\| \leq f(x)$,

$$
2\|x-y\|^{2}<\|w-x\|^{2} .
$$

Let $\widehat{z}$ be the diametric ball of $h$. Since $w$ is the closest vertex of $h$ to $y, \angle z y w \geq \pi / 2$. (Note that if $h$ is a subsegment, then $\angle z y w=\pi$.) This implies that $\|y-z\|^{2} \leq \operatorname{radius}(\widehat{z})^{2}-\|w-y\|^{2}$. So

$$
\begin{aligned}
\|x-z\|^{2} & =\|x-y\|^{2}+\|y-z\|^{2} \\
& \leq \operatorname{radius}(\widehat{z})^{2}+\|x-y\|^{2}-\|w-y\|^{2} \\
& =\operatorname{radius}(\widehat{z})^{2}+2\|x-y\|^{2}-\|w-x\|^{2}
\end{aligned}
$$

which is less than radius $(\widehat{z})^{2}$ by (4.11). But then rule 3 (SplitBalls) should split $h$ as $x$ lies inside $\widehat{z}$, a contradiction.

\section{$5 \quad$ Experimental results}

We experimented with a preliminary implementation of QMESH [19]. We protected sharp vertices with a vertex ball as demanded by the algorithm. We inserted all points where the edges intersect the vertex balls, but did not protect the shield subsegments with SOS. Apparently this does not cause any trouble for the datasets tested so far. It may very well be true that a more refined analysis can get rid of the SOS from the algorithm.

Figure 16 shows the results. Although we proved a bound of $2 \sqrt{2} /(1-\tan (\pi / 8))$ for the radius-edge ratio, we took $\rho_{0}=2.2$ for the experiments because Delaunay refinement often performs better in practice with $\rho_{0}$ close to 2 as in the standard Delaunay refinement [17] (also see the comment at the end of section 4.3.3). The tables below show that most of the tetrahedra have good radius-edge ratio. Tetrahedra with radius-edge ratio greater than 2.2 are shown in the second column of Figure 16. All of them lie near some small angle region. Third column of the figure shows the triangulation of the input polyhedron and some highlights to exhibit how the algorithm copes with small input angles.

\section{Discussions and open issues}

After the publication of a conference version of this paper, Pav and Walkington [15] presented an algorithm that can handle non-manifold boundaries at the expense of extra complications. All input edges are explicitly protected using an adaptive strategy. In the output mesh, most 

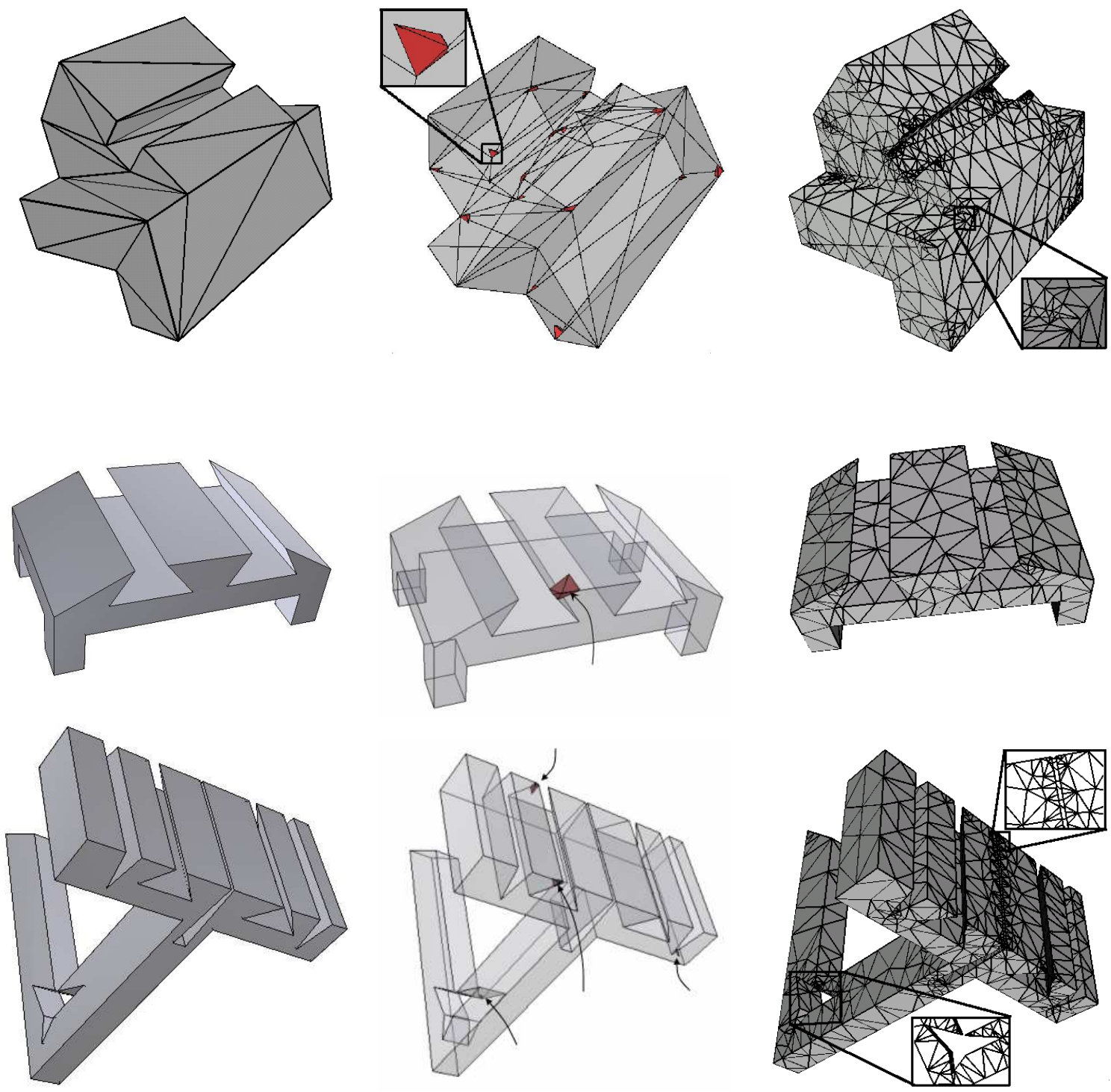

Figure 16: First column shows input polyhedra, ANCHOR, RAIL, WIPER from top to bottom. In the second column skinny tetrahedra that remain after QMESH are pointed out. Third column shows the triangulation of the polyhedra. 


\begin{tabular}{|c|c|c|}
\hline Model & \# input points & \# sharp elements \\
\hline Anchor & 28 & 27 \\
\hline Rail & 48 & 24 \\
\hline Wiper & 72 & 58 \\
\hline SimpleBox & 32 & 30 \\
\hline meshtest & 60 & 35 \\
\hline
\end{tabular}

\begin{tabular}{|c|c|c|c|c|}
\hline Model & \multirow{2}{*}{ \# points inserted } & \multicolumn{3}{|c|}{ \# tetrahedra with $R / \ell$} \\
\cline { 3 - 5 } & & $0.6-1.4$ & $1.4-2.2$ & $>2.2$ \\
\hline Anchor & 1260 & 3156 & 1254 & 35 \\
\hline Rail & 263 & 610 & 125 & 2 \\
\hline Wiper & 1121 & 2074 & 785 & 6 \\
\hline SimpleBox & 1334 & 3181 & 1131 & 40 \\
\hline meshtest & 300 & 798 & 286 & 0 \\
\hline
\end{tabular}

tetrahedra have bounded radius-edge ratio, except possibly those near the input edges. Since an implementation is not available yet, the practicality and effectiveness of this algorithm remain to be validated.

Although we proved that any remaining tetrahedra with poor radius-edge ratio lie near small input angles, we could not show that they are related to the smallest input angle. Pav and Walkington [15] also could not relate the radius-edge ratio of the skinny tetrahedra near the input edges to the smallest input angle. This is achieved in [5] though at the expense of added complications. It is an open problem to design a practical Delaunay meshing strategy that provides such a guarantee.

Also, questions remain if one can simplify the algorithm presented in this paper even further. Can we eliminate the bounding box? Can we eliminate the SOS step? Can one eliminate the doubling of the protecting balls at the end of CONFORM?

Finally, there are possibilities to extend the result of this paper in several directions. For example, handling non-manifold PLCs without making the algorithm much more complicated would be an important contribution. Bounded radius-edge ratio does not guarantee bounded aspect ratio for tetrahedra. Slivers are tetrahedra of bounded radius-edge ratio, but not of bounded aspect ratio. Actually, without eliminating slivers one cannot hope for any guarantee on the optimality of the size of the output. Eliminations of slivers with bounded domains is addressed in [3] though with the assumptions of no small angles. A combination of the techniques of this paper with those of [3] may give an algorithm for triangulating polyhedral spaces with guaranteed aspect ratio.

\section{Acknowledgments}

We thank the anonymous referees for their detailed and helpful comments. 


\section{References}

[1] M. Bern, D. Eppstein and J. Gilbert. Provably good mesh generation. J. Comput. Syst. Sci., 48 (1994), 384-409.

[2] C. Boivin and C. Ollivier-Gooch. Guaranteed-quality triangular mesh generation for domains with curved boundaries. Intl. J. Numer. Methods in Engineer., 55 (2002), 1185-1213.

[3] S.-W. Cheng and T. K. Dey. Quality meshing with weighted Delaunay refinement. SIAM J. Comput., 33 (2003), 69-93.

[4] S.-W. Cheng, T. K. Dey, H. Edelsbrunner, M. A. Facello and S.-H. Teng. Sliver exudation. J. ACM, 47 (2000), 883-904.

[5] S.-W. Cheng and S.-H. Poon. Graded conforming tetrahedralization with bounded radiusedge ratio. Proc. 14th Annu. ACM-SIAM Sympos. Discrete Algorithms (2003), 295-304.

[6] L. P. Chew. Guaranteed-quality triangular meshes. Report TR-98-983, Comput. Sci. Dept., Cornell Univ., Ithaca, New York, (1989).

[7] L. P. Chew. Guaranteed-quality Delaunay meshing in 3D. Proc. 13th Annu. ACM Sympos. Comput. Geom. (1997), 391-393.

[8] D. Cohen-Steiner, E. C. de Verdière and M. Yvinec. Conforming Delaunay triangulations in 3D. Proc. Annu. Sympos. Comput. Geom. (2002), 199-208.

[9] T. K. Dey, C. Bajaj and K. Sugihara. On good triangulations in three dimensions. Internat. J. Comput. Geom., 2 (1992), 75-95.

[10] H. Edelsbrunner. Geometry and Topology for Mesh Generation. Cambridge Univ. Press, England, 2001.

[11] X.-Y. Li and S.-H. Teng. Generating well-shaped Delaunay meshes in 3D. Proc. 12th. Annu. ACM-SIAM Sympos. Discrete Algorithm (2001), 28-37.

[12] G. L. Miller, D. Talmor, S.-H. Teng and N. Walkington. A Delaunay based numerical method for three dimensions: generation, formulation, and partition. Proc. 27th Annu. ACM Sympos. Theory Comput. (1995), 683-692.

[13] S. A. Mitchell and S. A. Vavasis. Quality mesh generation in higher dimensions. SIAM J. Comput., 29 (2000), 1334-1370.

[14] M. Murphy, D. M. Mount and C. W. Gable. A point placement strategy for conforming Delaunay tetrahedralization. Intl. J. Comput. Geom. Appl., 11 (2001), 669-682.

[15] S. Pav and N. Walkington. 3D robust Delaunay refinement. 14th Intl. Meshing Roundtable 2005.

[16] J. Ruppert. A Delaunay refinement algorithm for quality 2-dimensional mesh generation. J. Algorithms, 18 (1995), 548-585.

[17] J. R. Shewchuk. Tetrahedral mesh generation by Delaunay refinement. Proc. 14th Annu. ACM Sympos. Comput. Geom. (1998), 86-95. 
[18] J. R. Shewchuk. Mesh generation for domains with small angles. Proc. 16th Annu. Sympos. Comput. Geom. (2000), 1-10.

[19] http://www.cse.ohio-state.edu/ tamaldey/qualmesh.html. 